\title{
Regioselective Lithiation of Chiral 3-Acylamino-2-alkylquinazolin-4(3H)-ones: Application in Synthesis
}

\author{
Keith Smith, ${ }^{\text {a }}$ Gamal A. El-Hiti, ${ }^{\text {al }}$ Mohamed F. Abdel-Megeed ${ }^{b}$ \\ ${ }^{\mathrm{a}}$ Centre for Clean Chemistry, Department of Chemistry, University of Wales Swansea, Singleton Park, Swansea SA2 \\ 8PP, UK \\ ${ }^{\mathrm{b}}$ Department of Chemistry, Faculty of Science, Tanta University, Tanta 31527, Egypt \\ Fax: +44(1792)295261 \\ E-mail: k.smith@swansea.ac.uk \\ Received:
}

\begin{abstract}
Reaction of 3-amino-2-alkylquinazolin-4(3H)-ones with several chiral acid chlorides was found to be dependent on the molar proportions. When a 1:1 molar mixture was heated under reflux, the corresponding 3-(diacylamino)- derivatives were obtained in poor yields. However, when a 2:1 molar mixture was reacted in refluxing toluene, the 3 -acylamino- derivatives were obtained in good yields based on the acid chloride. Lithiation of the 3-acylamino-2-alkylquinazolin-4(3H)-ones was achieved by the use of LDA in anhydrous THF at $-78^{\circ} \mathrm{C}$ and the reaction was regioselective at the carbon $\alpha$ to position 2 of the quinazolin$4(3 H)$-one moiety. The dilithio reagents thus obtained reacted with electrophiles to give the corresponding 2-substituted derivatives in very good yields. The NMR spectra of the products show the expected diastereotopism for all the $\mathrm{CH}_{2}$ groups and provide evidence for long-range asymmetric induction.
\end{abstract}

Key words: lithiation, quinazolin-4(3H)-ones, dilithio reagent, asymmetric induction, diastereotopism

Directed metallation $^{2,3}$ has found wide use in regioselective introduction of functional substituents into aromatic and heterocyclic compounds. However, there are relatively few examples concerning directed metallation of quinazolin-4(3H)-ones to afford more complex substituted derivatives. $^{4-13}$ Compounds possessing this ring system are of interest because they show a variety of biological activities. $^{14}$ In continuation of our own interest in the use of lithiation reactions for organic synthesis, ${ }^{15}$ we have demonstrated the lithiation of various quinazolin-4(3H)-ones, ${ }^{6-8}$ including simple 3-acylamino derivatives. $^{9,10}$ We now report a study of the scope of the lithiation reaction for more complex 3-acylamino-2alkylquinazolin-4(3H)-ones containing chiral acyl units. We have been able to achieve regioselective lithiation of a range of such quinazolin-4(3H)-ones by the use of LDA, and the synthesis of more complex 2-substituted derivatives in good yields. The products also show evidence of long-range asymmetric induction.

The first stage of this study required the synthesis of representative chiral 3-acylamino-2-alkylquinazolin$4(3 H)$-ones. Initially, reactions of 3-amino-2ethylquinazolin-4(3H)-one $(\mathbf{1})^{16}$ with chiral but racemic acid chlorides in the presence of triethylamine at $0{ }^{\circ} \mathrm{C}$ or room temperature were attempted. However, 1 was recovered unchanged, suggesting that no reaction had occurred under these conditions. A series of experiments was conducted in which the reaction conditions were varied in an attempt to produce the corresponding acylamino derivatives. When a 1:1 molar mixture of $\mathbf{1}$ and an acid chloride in the presence of triethylamine was heated under reflux in anhydrous toluene for $1 \mathrm{~h}$ the corresponding diacylamino derivative $\mathbf{2}$ or $\mathbf{3}$ was obtained in $41 \%$ or $25 \%$ isolated yield, respectively (Scheme 1). The monoacylamino derivative ( $\mathbf{5}$ or $\mathbf{8}$ ) was formed in only low yield and a substantial quantity of unreacted 1 remained. 


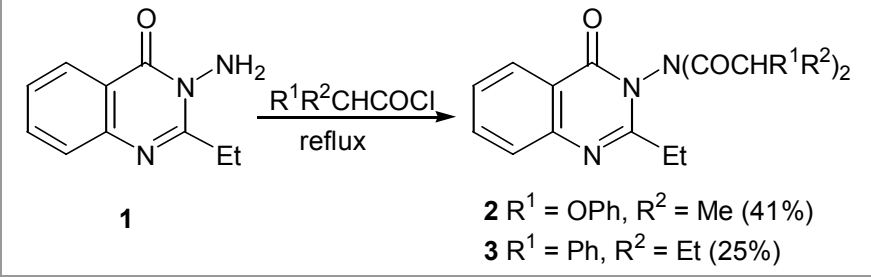

Scheme 1

Product 2 appeared from its NMR spectra as a mixture of two racemic diastereoisomers in unequal proportions, while product 3 appeared from its ${ }^{1} \mathrm{H}$ NMR spectrum as a pair of racemic diastereoisomers in approximately equal proportions. The ${ }^{1} \mathrm{H}$ NMR spectrum of $\mathbf{3}$ showed diastereotopism for the hydrogens of the $\mathrm{CH}_{2}$ groups at position 2 and in the acyl units.

In a modified procedure for the attempted synthesis of a chiral 3-acylamino-2-alkylquinazolin-4(3H)-one a 2:1 molar mixture of 3-amino-2-ethylquinazolin-4(3H)-one (1) and 2-phenoxypropanoyl chloride was heated under reflux in anhydrous toluene for $1 \mathrm{~h}$ in the presence of triethylamine. Work-up gave the desired product $\mathbf{5}$ in $78 \%$ yield. Therefore, these conditions were applied in the synthesis of a range of products (5-10, Scheme 2) derived from both 1 and 3-amino-2-propylquinazolin$4(3 H)$-one $(4)^{17}$ with a variety of acid chlorides. This procedure afforded products 5-10 in very good yields based on the acid chloride (Table 1).

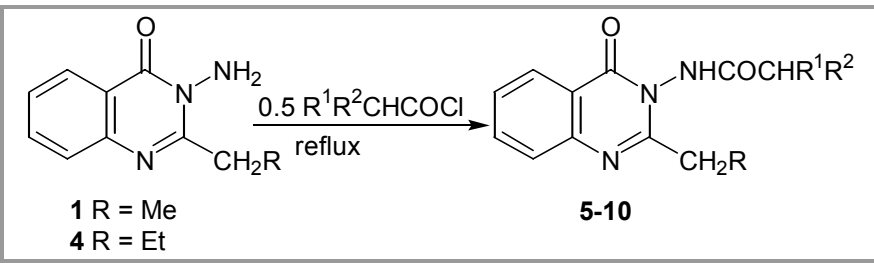

Scheme 2
Table 1 Synthesis of products 5-10 According to Scheme 2

\begin{tabular}{lllll} 
Compound & $\mathrm{R}$ & $\mathrm{R}^{1}$ & $\mathrm{R}^{2}$ & Yield (\%) $^{a}$ \\
\hline $\mathbf{5}$ & $\mathrm{Me}$ & $\mathrm{Me}$ & $\mathrm{OPh}$ & 78 \\
$\mathbf{6}$ & $\mathrm{Me}$ & $\mathrm{Me}$ & 1-Naphthyl & 74 \\
$\mathbf{7}$ & $\mathrm{Me}$ & $\mathrm{Et}$ & $\mathrm{OPh}$ & 80 \\
$\mathbf{8}$ & $\mathrm{Me}$ & $\mathrm{Et}$ & $\mathrm{Ph}$ & 79 \\
$\mathbf{9}$ & $\mathrm{Et}$ & $\mathrm{Me}$ & $\mathrm{OPh}$ & 90 \\
$\mathbf{1 0}$ & $\mathrm{Et}$ & $\mathrm{Et}$ & $\mathrm{OPh}$ & 93
\end{tabular}

${ }^{a}$ Yields of isolated, purified products based on acid chloride added.

Products 5-10 appear from their NMR spectra as mixtures of two diastereoisomers in unequal proportions, due to restricted rotation around the N-N axis. ${ }^{18}$ The spectra also showed diastereotopism for the $\mathrm{CH}_{2}$ protons of the ethyl and propyl groups (see experimental section for details). The ${ }^{1} \mathrm{H}$ NMR spectra were temperature dependent and showed some evidence of equilibration at 55-60 ${ }^{\circ} \mathrm{C}$ in $\mathrm{CDCl}_{3}$. In DMSO- $d_{6}$, the spectra showed a single set of signals at $150{ }^{\circ} \mathrm{C}$ in all cases.

In order to introduce more complex substituents at position 2, lithiations of 5-10 were carried out. Lithiation was achieved by the use of LDA in anhydrous THF at $-78{ }^{\circ} \mathrm{C}$ under nitrogen and the reaction was regioselective at the carbon $\alpha$ to position 2 of the quinazolin4(3H)-one moiety. Addition of one equivalent of LDA presumably produced the monolithio reagents $\mathbf{1 1}$, which were converted into the dilithio reagents $\mathbf{1 2}$ on addition of a second equivalent of LDA. Reactions of the dilithio reagents 12 with several electrophiles (benzophenone, benzaldehyde, acetophenone, 2-butanone) afforded the corresponding 2-substituted quinozolin-4(3H)-ones 1327 (Scheme 3) in very good yields (Table 2) following column chromatography. 
The NMR spectra of all products containing a $\mathrm{CH}_{2}$ group either in the side chain at position 2 or in the acylamino group (compounds 17-27) showed the expected diastereotopism for the $\mathrm{CH}_{2}$ protons. In addition, the spectra (of the total product obtained following chromatography to remove coloured impurities) revealed the presence of diastereoisomers.

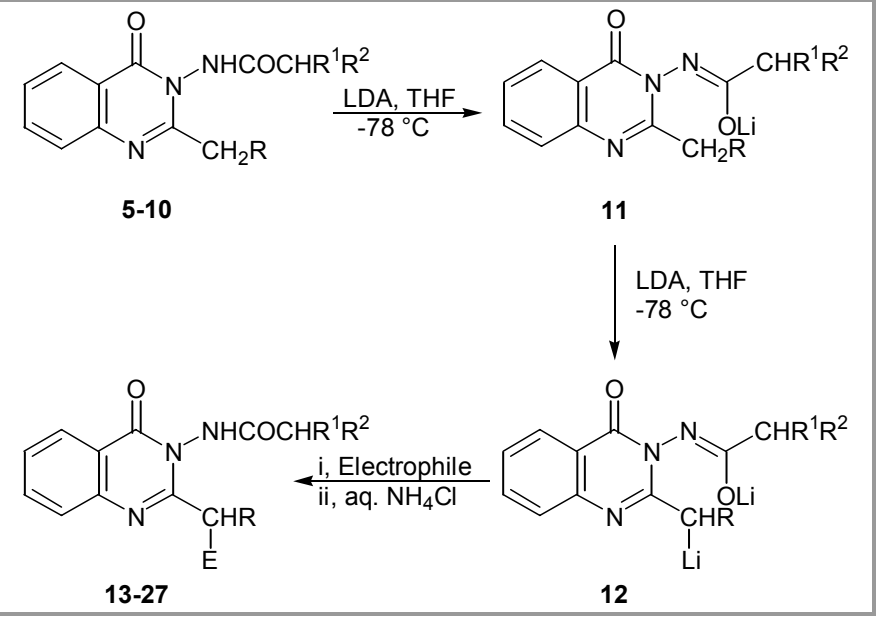

Scheme 3

Table 2 Synthesis of Products 13-27 According to Scheme 3

\begin{tabular}{|c|c|c|c|c|c|}
\hline Compound & $\mathrm{R}$ & $\mathrm{R}^{1}$ & $\mathrm{R}^{2}$ & E & Yield $(\%)^{a}$ \\
\hline 13 & $\mathrm{Me}$ & $\mathrm{Me}$ & $\mathrm{OPh}$ & $\mathrm{Ph}_{2} \mathrm{C}(\mathrm{OH})$ & 80 \\
\hline 14 & $\mathrm{Me}$ & $\mathrm{Me}$ & $\mathrm{OPh}$ & $\mathrm{PhCH}(\mathrm{OH})$ & 82 \\
\hline 15 & $\mathrm{Me}$ & $\mathrm{Me}$ & $\mathrm{OPh}$ & $\mathrm{PhC}(\mathrm{OH}) \mathrm{Me}$ & 78 \\
\hline 16 & $\mathrm{Me}$ & $\mathrm{Me}$ & 1-naphthyl & $\mathrm{Ph}_{2} \mathrm{C}(\mathrm{OH})$ & 79 \\
\hline 17 & $\mathrm{Me}$ & Et & $\mathrm{OPh}$ & $\mathrm{Ph}_{2} \mathrm{C}(\mathrm{OH})$ & 76 \\
\hline 18 & $\mathrm{Me}$ & Et & $\mathrm{OPh}$ & $\mathrm{PhCH}(\mathrm{OH})$ & 80 \\
\hline 19 & $\mathrm{Me}$ & Et & $\mathrm{OPh}$ & $\mathrm{PhC}(\mathrm{OH}) \mathrm{Me}$ & 79 \\
\hline 21 & $\mathrm{Me}$ & Et & $\mathrm{Ph}$ & $\mathrm{EtC}(\mathrm{OH}) \mathrm{Me}$ & 75 \\
\hline 22 & $\mathrm{Et}$ & $\mathrm{Me}$ & $\mathrm{OPh}$ & $\mathrm{Ph}_{2} \mathrm{C}(\mathrm{OH})$ & 88 \\
\hline 23 & Et & $\mathrm{Me}$ & $\mathrm{OPh}$ & $\mathrm{PhCH}(\mathrm{OH})$ & 76 \\
\hline 24 & Et & $\mathrm{Me}$ & $\mathrm{OPh}$ & $\mathrm{PhC}(\mathrm{OH}) \mathrm{Me}$ & 80 \\
\hline 25 & Et & Et & $\mathrm{OPh}$ & $\mathrm{Ph}_{2} \mathrm{C}(\mathrm{OH})$ & 90 \\
\hline 26 & Et & Et & $\mathrm{OPh}$ & $\mathrm{PhCH}(\mathrm{OH})$ & 80 \\
\hline 27 & Et & Et & $\mathrm{OPh}$ & $\mathrm{PhC}(\mathrm{OH}) \mathrm{Me}$ & 78 \\
\hline
\end{tabular}

The number of diastereoisomers of the product expected when benzophenone is used as the electrophile is four. Indeed, it was found that the NMR spectra of products 13, 22 and 25 showed a mixture of the expected four diastereoisomers in unequal proportions. However, the NMR spectra of products $\mathbf{1 7}$ and $\mathbf{2 0}$ showed the predominant presence of two substantial diastereoisomers in unequal proportions, while in the case of product $\mathbf{1 6}$, there were two major diastereoisomers in approximately equal proportions.
Product 13 was separated into two fractions by crystallisation. The first fraction (13a, 60\%) showed the presence of a single diastereoisomer. The x-ray crystallography of this compound (Figure 1) indicated the presence of one THF molecule for each molecule of 13a. The THF appeared to be hydrogen-bonded to the $\mathrm{NH}$ proton of the acylamino group. The crystal structure also showed that this isomer was 2-((1S*)-2,2-diphenyl-2hydroxy-1-methyl)-3-((2R*)-2-

phenoxypropionylamino)quinazolin-4(3H)-one with $\left(R_{\mathrm{a}}{ }^{*}\right)$ configuration about the $\mathrm{N}-\mathrm{N}$ axis. The second 
fraction (40\%) showed the presence of all four diastereoisomers, with $13 \mathbf{a}$ being a minor component.

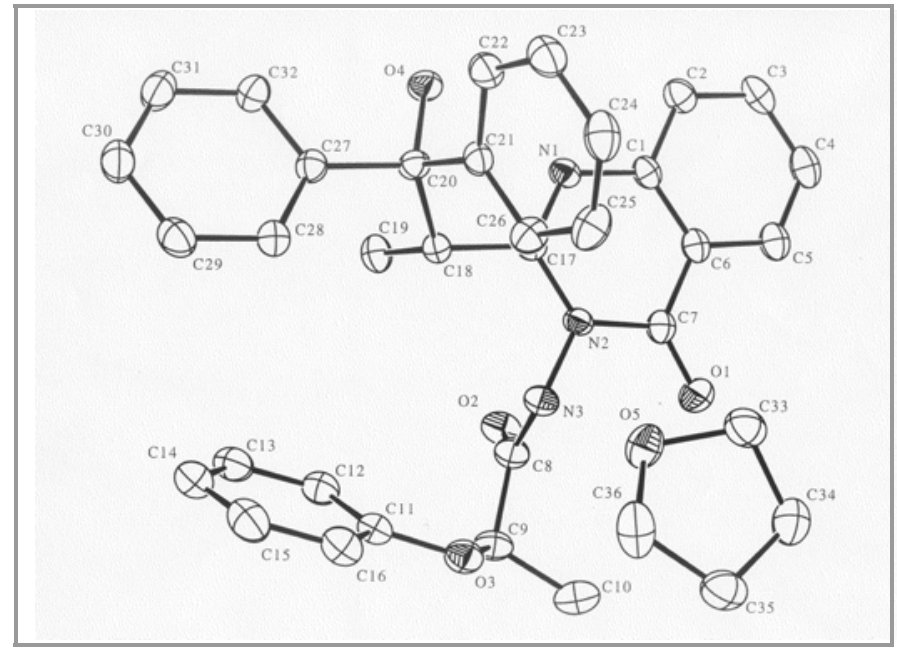

Figure $1 \mathrm{X}$-ray crystal structure of 13a with numbered crystallographic atoms

Variable temperature ${ }^{1} \mathrm{H}$ NMR of 13a up to $60{ }^{\circ} \mathrm{C}$ in $\mathrm{CDCl}_{3}$ showed only minimal line broadening. However, a sample of 13a left at room temperature for many months, showed a ${ }^{1} \mathrm{H}$ NMR spectrum corresponding to a mixture of 13a and one other diastereoisomer, presumably the one involving the alternative stereochemistry $\left(S_{\mathrm{a}}^{*}\right)$ about the N-N axis. This enabled identification of the two diastereoisomers having the same configuration at the new asymmetric carbon atom. The ${ }^{1} \mathrm{H}$ NMR spectrum of the second fraction (mixture) showed that these two components accounted for about $18 \%$ of the fraction, i.e. about $7 \%$ of the total product, bringing the total amount of the isomers having that stereochemistry at the new asymmetric carbon atom to around $67 \%$. The other two diastereoisomers, having the alternative configuration at the new asymmetric carbon atom, accounted for the remaining $33 \%$ or so. Therefore, the new asymmet- ric centre is formed with a 2:1 stereoselectivity. Although this selectivity is relatively small, it is nevertheless noteworthy, since it represents a long-range asymmetric inductive effect (the two asymmetric carbon atoms have a 1,6-relationship).

For product 17, the two major diastereoisomers both had the same configuration at the new asymmetric carbon atom. This was deduced by the fact that the newly created $\mathrm{CH}$ signals for those two isomers in the ${ }^{1} \mathrm{H}$ NMR spectrum began to coalesce at $60{ }^{\circ} \mathrm{C}$. Furthermore, the chemical shift positions were virtually identical to those for 13a and its N-N isomer. The two major diastereoisomers accounted for about $80 \%$ of the total, leading to the conclusion that the long-range asymmetric induction produced a ratio of configurations at the new centre of ca. $4: 1$.

For products $\mathbf{1 6}$ and $\mathbf{2 0}$, the NMR signals were more difficult to resolve and it was therefore much more difficult to identify the individual diastereoisomers with any confidence, although it was clear, particularly from the ${ }^{13} \mathrm{C}$ NMR spectra, that two diastereoisomers were predominant. At $60{ }^{\circ} \mathrm{C}$ the ${ }^{1} \mathrm{H}$ NMR spectra showed some line broadening, but it was still not clear whether the two major isomers had different configurations at the new asymmetric carbon atoms or whether they differed about the N-N axis.

When an unsymmetrical carbonyl compound (benzaldehyde, acetophenone or 2-butanone) is used as electrophile, the number of diastereoisomers expected to be 
formed is eight. The ambient temperature NMR spectra of 21 (2-butanone used as the electrophile) showed the presence of at least seven racemic diastereoisomers in unequal proportions. However, the ambient temperature NMR spectra of products $14,15,18,19$ and 24 showed mixtures of no more than four substantial diastereoisomers in unequal proportions. Furthermore, for this group of products, the ${ }^{1} \mathrm{H}$ NMR spectra recorded at $100{ }^{\circ} \mathrm{C}$ showed the presence of only two major diastereoisomers in unequal proportions, which indicates equilibration via rotation about the $\mathrm{N}-\mathrm{N}$ axis. In the case of products $\mathbf{2 3}$, 26 and 27 the ambient temperature NMR spectra showed mixtures of only two significant diastereoisomers in unequal proportions, which indicates a considerable long-range asymmetric induction. Unfortunately, an attempt to bring about equilibration in these cases by recording the ${ }^{1} \mathrm{H}$ NMR at $150{ }^{\circ} \mathrm{C}$ resulted in some decomposition to give bezaldehyde or acetophenone.

In conclusion, lithiation of chiral 3-acylamino-2alkylquinazolin-4(3H)-ones followed by reactions with carbonyl compounds is useful for the elaboration of more complex 2-substituted 3-acylaminoquinazolin$4(3 H)$-ones, and in some cases gives considerable longrange asymmetric induction at the newly created asymmetric centre(s). This opens up possibilities for novel synthetic approaches to certain types of chiral compounds, which we intend to investigate.

Melting point determinations were performed by the open capillary method using a Gallenkamp melting point apparatus and are reported uncorrected. The laboratories of the University of Wales Cardiff carried out micro- analyses. ${ }^{1} \mathrm{H}$ and ${ }^{13} \mathrm{C}$ NMR spectra were recorded on a Bruker AC400 spectrometer operating at $400 \mathrm{MHz}$ for ${ }^{1} \mathrm{H}$ and $100 \mathrm{MHz}$ for ${ }^{13} \mathrm{C}$ measurements. Chemical shifts are reported relative to tetramethylsilane. Assignments of signals are based on coupling patterns and expected chemical shift values and have not been rigorously confirmed. Signals with similar characteristics might be interchanged. Low-resolution mass spectra were recorded on a VG 12-253 spectrometer, electron impact (EI) at $70 \mathrm{eV}$ and chemical ionization (CI) by the use of ammonia as ionization gas. FAB mass spectra were recorded on a VG-Autospec instrument. Accurate mass data were obtained on a VG ZAB-E instrument. Column chromatography was carried out using Merck Kieselgel 60 (230-400 mesh). Lithium diisopropylamide (LDA) and other chemicals were obtained from Aldrich Chemical Company and used without further purification. THF was distilled from sodium benzophenone ketyl. Other solvents were purified by standard procedures. ${ }^{19,20}$

\section{3-(Diacylamino)-2-ethylquinazolin-4(3H)-ones (2) and (3); General Procedure}

To a stirred mixture of 1 (1.9 g, $10 \mathrm{mmol})$ and $\mathrm{Et}_{3} \mathrm{~N}(5$ $\mathrm{mL})$ in anhydrous toluene $(20 \mathrm{~mL})$, was added a solution of the appropriate acid chloride $(11 \mathrm{mmol})$ in anhydrous toluene $(5 \mathrm{~mL})$. The mixture was heated under reflux for $30 \mathrm{~min}$, allowed to cool, washed with sat. aq. $\mathrm{NaHCO}_{3}$ $(2 \times 10 \mathrm{~mL})$ and $\mathrm{H}_{2} \mathrm{O}(2 \times 15 \mathrm{~mL})$, dried $\left(\mathrm{MgSO}_{4}\right)$, and evaporated under reduced pressure. The residue obtained was purified by column chromatography on silica gel $\left(\mathrm{Et}_{2} \mathrm{O}\right.$-hexane, 1:4) to give $\mathbf{2}$ or $\mathbf{3}$ as a white powder.

\section{2-Ethyl-3-[di(2-phenoxypropionyl)amino]quinazolin- 4(3H)-one (2)}

mp 104-107 ${ }^{\circ} \mathrm{C}$; pair of racemic diastereoisomers; $2 \mathbf{a} / \mathbf{2} \mathbf{b}$ $=3: 5\left({ }^{1} \mathrm{H}\right.$ NMR).

FAB-MS: $m / z(\%)=508\left(\mathrm{M}^{+}+\mathrm{Na}, 20\right), 486\left(\mathrm{MH}^{+}, 67\right)$, 392 (10), 338 (31), 216 (33), 121 (100).

HRMS: $m / z$ calcd for $\mathrm{C}_{28} \mathrm{H}_{28} \mathrm{~N}_{3} \mathrm{O}_{5}\left(\mathrm{MH}^{+}\right)$: 486.2029 ; found: 486.2017 .

\section{Compound 2a}

${ }^{1} \mathrm{H}$ NMR $\left(\mathrm{CDCl}_{3}\right): \delta=8.17(\mathrm{dd}, J=8,1 \mathrm{~Hz}, 1 \mathrm{H}, \mathrm{H} 5)$, 7.69 (app. dt, $J=8,1 \mathrm{~Hz}, 1 \mathrm{H}, \mathrm{H} 7), 7.48$ (d, $J=8 \mathrm{~Hz}, 1$ H, H8), 7.41 (app. dt, $J=8,1 \mathrm{~Hz}, 1 \mathrm{H}, \mathrm{H} 6$ ), 7.26-6.74 $(\mathrm{m}, 10 \mathrm{H}, 2 \mathrm{OPh}), 5.43$ (q, $J=6.5 \mathrm{~Hz}, 2 \mathrm{H}, 2 \mathrm{CH}), 2.27$ $\left(\mathrm{q}, J=7 \mathrm{~Hz}, 2 \mathrm{H}, \mathrm{CH}_{2}\right), 1.52(\mathrm{~d}, J=6.5 \mathrm{~Hz}, 6 \mathrm{H}, 2$ $\left.\mathrm{CH}_{3} \mathrm{CH}\right), 1.09$ (t, $J=7 \mathrm{~Hz}, 3 \mathrm{H}, \mathrm{CH}_{3} \mathrm{CH}_{2}$ ).

${ }^{13} \mathrm{C} \mathrm{NMR}\left(\mathrm{CDCl}_{3}\right): \delta=172.6(\mathrm{~s}, \mathrm{C}=\mathrm{O}), 160.2(\mathrm{~s}, \mathrm{C} 4)$, 157.1 (s, C2), $156.8(\mathrm{~s}, \mathrm{C} 1$ of $2 \mathrm{OPh}), 146.6$ (s, C8a), 135.2 (d, C7), 129.7 (d, C3 of OPh), 127.6 (d, C5), 127.0 (d, C6), 126.8 (d, C8), 122.4 (d, C4 of OPh), 120.3 (s, $\mathrm{C} 4 \mathrm{a}), 115.3$ (d, C2 of OPh), $72.9(\mathrm{~d}, \mathrm{CH}), 25.5\left(\mathrm{t}, \mathrm{CH}_{2}\right)$, 17.8 (q, $\left.\mathrm{CH}_{3} \mathrm{CH}\right), 9.7$ (q, $\mathrm{CH}_{3} \mathrm{CH}_{2}$ ).

\section{Compound 2 b}


${ }^{1} \mathrm{H}$ NMR $\left(\mathrm{CDCl}_{3}\right): \delta=8.17(\mathrm{dd}, J=8,1 \mathrm{~Hz}, 1 \mathrm{H}, \mathrm{H} 5)$, 6.69 (app. dt, $J=8,1 \mathrm{~Hz}, 1 \mathrm{H}, \mathrm{H} 7), 7.53$ (d, $J=8 \mathrm{~Hz}, 1$ H, H8), 7.41 (app. dt, $J=8,1 \mathrm{~Hz}, 1 \mathrm{H}, \mathrm{H6}$ ), 7.26-6.60 $(\mathrm{m}, 10 \mathrm{H}, 2 \mathrm{OPh}), 5.83(\mathrm{q}, J=6.5 \mathrm{~Hz}, 2 \mathrm{H}, 2 \mathrm{CH}), 2.31$ (q, $\left.J=7 \mathrm{~Hz}, 2 \mathrm{H}, \mathrm{CH}_{2}\right), 1.64(\mathrm{~d}, J=6.5 \mathrm{~Hz}, 6 \mathrm{H}, 2$ $\left.\mathrm{CH}_{3} \mathrm{CH}\right), 1.18\left(\mathrm{t}, J=7 \mathrm{~Hz}, 3 \mathrm{H}, \mathrm{CH}_{3} \mathrm{CH}_{2}\right)$.

${ }^{13} \mathrm{C} \mathrm{NMR}\left(\mathrm{CDCl}_{3}\right): \delta=171.8(\mathrm{~s}, \mathrm{C}=\mathrm{O}), 160.2(\mathrm{~s}, \mathrm{C} 4)$, 157.2 (s, C2), 155.7 (s, C1 of OPh), 146.6 (s, C8a), 135.3 (d, C7), 129.6 (d, C3 of OPh), 127.7 (d, C5), 127.1 (d, C6), 126.8 (d, C8), 122.0 (d, C4 of OPh), 120.3 (s, $\mathrm{C} 4 \mathrm{a}), 115.0$ (d, $\mathrm{C} 2$ of $\mathrm{OPh}), 73.4(\mathrm{~d}, \mathrm{CH}), 25.6\left(\mathrm{t}, \mathrm{CH}_{2}\right)$, 17.8 (q, $\left.\mathrm{CH}_{3} \mathrm{CH}\right), 9.8$ (q, $\mathrm{CH}_{3} \mathrm{CH}_{2}$ ).

\section{2-Ethyl-3-[di(2-phenylbutyryl)amino]quinazolin- 4(3H)-one (3)}

mp 155-156 ${ }^{\circ} \mathrm{C}$; pair of racemic diastereoisomers (NMR).

FAB-MS: $m / z(\%)=504\left(\mathrm{M}^{+}+\mathrm{Na}, 23\right), 482\left(\mathrm{MH}^{+}, 69\right)$, 336 (86), 216 (36), 190 (36), 119 (100).

HRMS: $m / z$ calcd for $\mathrm{C}_{30} \mathrm{H}_{32} \mathrm{~N}_{3} \mathrm{O}_{3}\left(\mathrm{MH}^{+}\right)$: 482.2444; found: 482.2459 .

${ }^{1} \mathrm{H}$ NMR $\left(\mathrm{CDCl}_{3}\right): \delta=8.30(\mathrm{dd}, J=8,1 \mathrm{~Hz}, 1 \mathrm{H}, \mathrm{H} 5)$, 7.80 (app. dt, $J=8,1 \mathrm{~Hz}, 1 \mathrm{H}, \mathrm{H} 7), 7.68$ (d, $J=8 \mathrm{~Hz}, 1$ H, H8), 7.50 (app. dt, $J=8,1 \mathrm{~Hz}, 1 \mathrm{H}, \mathrm{H} 6), 7.33$ (m, 4 $\mathrm{H}, \mathrm{H} 3$ of $2 \mathrm{Ph}), 7.12(\mathrm{~m}, 4 \mathrm{H}, \mathrm{H} 2$ of $2 \mathrm{Ph}), 6.70(\mathrm{~d}, J=$ $7.5 \mathrm{~Hz}, 2 \mathrm{H}, \mathrm{H} 4$ of $2 \mathrm{Ph}), 4.98,3.23(2 \mathrm{t}, J=7 \mathrm{~Hz}, 2 \mathrm{H}, 2$ $\mathrm{CH}), 2.24,2.14\left(2 \mathrm{dq}, J=14,7 \mathrm{~Hz}, 2 \mathrm{H}, \mathrm{CH}_{2} \mathrm{CH}_{3}\right), 1.85-$ $1.68\left(\mathrm{~m}, 2 \mathrm{H}, \mathrm{CH}_{2} \mathrm{CH}\right), 1.30-0.93\left(\mathrm{~m}, 2 \mathrm{H}, \mathrm{CH}_{2} \mathrm{CH}\right)$, $0.93,0.80\left(2 \mathrm{t}, J=7 \mathrm{~Hz}, 6 \mathrm{H}, 2 \mathrm{CH}_{3} \mathrm{CH}_{2} \mathrm{CH}\right), 0.57$ (t, $J=$ $\left.7 \mathrm{~Hz}, 3 \mathrm{H}, \mathrm{CH}_{3} \mathrm{CH}_{2}\right)$.

${ }^{13} \mathrm{C}$ NMR $\left(\mathrm{CDCl}_{3}\right): \delta=174.2,173.9(2 \mathrm{~s}, \mathrm{C}=\mathrm{O}), 160.4$ (s, C4), 158.2 (s, C2), 147.0 (s, C8a), 137.8, 136.6 (2 s, $\mathrm{C} 1$ of Ph), 135.3 (d, C7), 129.0, 128.9 (2 d, C3 of Ph), 128.5, 128.4 ( $2 \mathrm{~d}, \mathrm{C} 4$ of $\mathrm{Ph}), 127.8,127.7$ ( $2 \mathrm{~d}, \mathrm{C} 2$ of $\mathrm{Ph}), 127.5$ (d, C5), 127.4 (d, C6), 126.8 (d, C8), 120.5 (s, C4a), 54.0, 53.5 (2 d, CH), 27.7, $27.0\left(2 \mathrm{t}, \mathrm{CH}_{2} \mathrm{CH}\right), 24.3$ (t, $\left.\mathrm{CH}_{3} \mathrm{CH}_{2}\right), 11.9,11.7\left(2 \mathrm{q}, \mathrm{CH}_{3} \mathrm{CH}_{2} \mathrm{CH}\right), 9.5$ (q, $\mathrm{CH}_{3} \mathrm{CH}_{2}$ ).

\section{3-Acylamino-2-alkylquinazolin-4(3H)-ones General Procedure}

(5-10);

To a stirred mixture of 1 or $\mathbf{4}(10 \mathrm{mmol})$ and $\mathrm{Et}_{3} \mathrm{~N}$ (3 $\mathrm{mL})$ in anhydrous toluene $(20 \mathrm{~mL})$, was added a solution of the appropriate acid chloride $(5 \mathrm{mmol})$ in anhydrous toluene $(5 \mathrm{~mL})$. The mixture was heated under reflux for $30 \mathrm{~min}$, allowed to cool, washed with sat. aq. $\mathrm{NaHCO}_{3}$ $(2 \times 10 \mathrm{~mL})$ and $\mathrm{H}_{2} \mathrm{O}(2 \times 15 \mathrm{~mL})$, dried $\left(\mathrm{MgSO}_{4}\right)$, and evaporated under reduced pressure. The residue obtained was purified by column chromatography on silica gel ( $\mathrm{Et}_{2} \mathrm{O}$-hexane, $\left.1: 4\right)$ to give the corresponding 3-acylamino derivatives 5-10. The yields obtained are recorded in Table 1.

\section{2-Ethyl-3-(2-phenoxypropionylamino)quinazolin- 4(3H)-one (5)}

mp 90-92 ${ }^{\circ} \mathrm{C} ; \mathbf{5} \mathbf{a} / \mathbf{5 b}=1: 2\left({ }^{1} \mathrm{H}\right.$ NMR).

Anal. Calcd for $\mathrm{C}_{19} \mathrm{H}_{19} \mathrm{~N}_{3} \mathrm{O}_{3}: \mathrm{C}, 67.65 ; \mathrm{H}, 5.64 ; \mathrm{N}$, 12.46. Found: $\mathrm{C}, 67.6 ; \mathrm{H}, 5.6 ; \mathrm{N}, 12.6$.

EI-MS: $m / z(\%)=337\left(\mathrm{M}^{+}, 22\right), 244(13), 216(100)$, 173 (32), 130 (34), 121 (81), 77 (99).

HRMS: $m / z$ calcd for $\mathrm{C}_{19} \mathrm{H}_{19} \mathrm{~N}_{3} \mathrm{O}_{3}\left(\mathrm{M}^{+}\right)$: 337.1426; found: 337.1426.

\section{Compound 5a}

${ }^{1} \mathrm{H} \mathrm{NMR}\left(\mathrm{CDCl}_{3}\right): \delta=8.73$ (s, exch., $\left.1 \mathrm{H}, \mathrm{NH}\right), 8.16(\mathrm{dd}$, $J=8,1 \mathrm{~Hz}, 1 \mathrm{H}, \mathrm{H} 5), 7.72$ (m, $1 \mathrm{H}, \mathrm{H} 7), 7.64$ (d, $J=8$

$\mathrm{Hz}, 1 \mathrm{H}, \mathrm{H} 8$ ), 7.41 (app. dt, $J=8,1 \mathrm{~Hz}, 1 \mathrm{H}, \mathrm{H} 6$ ), 7.35 $(\mathrm{t}, J=7.5 \mathrm{~Hz}, 2 \mathrm{H}, \mathrm{H} 3$ of OPh), $7.07(\mathrm{t}, J=7.5 \mathrm{~Hz}, 2 \mathrm{H}$, $\mathrm{H} 2$ of OPh), $6.99(\mathrm{~d}, J=7.5 \mathrm{~Hz}, 1 \mathrm{H}, \mathrm{H} 4 \mathrm{of} \mathrm{OPh}), 4.97$ (q, $J=7 \mathrm{~Hz}, 1 \mathrm{H}, \mathrm{CH}), 2.44(2 \mathrm{dq}, J=15,7.5 \mathrm{~Hz}, 2 \mathrm{H}$, $\left.\mathrm{CH}_{2}\right), 1.81\left(\mathrm{~d}, J=7 \mathrm{~Hz}, 3 \mathrm{H}, \mathrm{CH}_{3} \mathrm{CH}\right), 1.06(\mathrm{t}, J=7.5$ $\mathrm{Hz}, 3 \mathrm{H}, \mathrm{CH}_{3} \mathrm{CH}_{2}$ ).

${ }^{13} \mathrm{C}$ NMR $\left(\mathrm{CDCl}_{3}\right): \delta=172.8(\mathrm{~s}, \mathrm{C}=\mathrm{O}), 159.9$ (s, C4), 158.5 (s, C2), 156.8 (s, C1 of OPh), $147.0(\mathrm{~s}, \mathrm{C} 8 \mathrm{a})$, 134.8 (d, C7), 129.9 (d, C3 of OPh), 127.5 (d, C5), 126.9 (d, C6), 126.7 (d, C8), 122.5 (d, C4 of OPh), 120.6 (s, C4a), 115.2 (d, C2 of OPh), $74.7(\mathrm{~d}, \mathrm{CH}), 27.0\left(\mathrm{t}, \mathrm{CH}_{2}\right)$, 18.8 (q, $\mathrm{CH}_{3} \mathrm{CH}$ ), 10.5 (q, $\mathrm{CH}_{3} \mathrm{CH}_{2}$ ).

\section{Compound 5b}

${ }^{1} \mathrm{H}$ NMR $\left(\mathrm{CDCl}_{3}\right): \delta=8.93$ (s, exch., $\left.1 \mathrm{H}, \mathrm{NH}\right), 8.12(\mathrm{~d}$, $J=8 \mathrm{~Hz}, 1 \mathrm{H}, \mathrm{H} 5), 7.72(\mathrm{~m}, 1 \mathrm{H}, \mathrm{H} 7), 7.64(\mathrm{~d}, J=8 \mathrm{~Hz}$, $1 \mathrm{H}, \mathrm{H} 8$ ), 7.41 (app. dt, $J=8,1 \mathrm{~Hz}, 1 \mathrm{H}, \mathrm{H} 6), 7.35$ (t, $J=$ $7.5 \mathrm{~Hz}, 2 \mathrm{H}, \mathrm{H} 3 \mathrm{of} \mathrm{OPh}), 7.07(\mathrm{t}, J=7.5 \mathrm{~Hz}, 2 \mathrm{H}, \mathrm{H} 2$ of $\mathrm{OPh}), 6.99(\mathrm{~d}, J=7.5 \mathrm{~Hz}, 1 \mathrm{H}, \mathrm{H} 4 \mathrm{of} \mathrm{OPh}), 4.95(\mathrm{q}, J=$ $7 \mathrm{~Hz}, 1 \mathrm{H}, \mathrm{CH}), 2.81$ (2 dq, $J=15,7.5 \mathrm{~Hz}, 2 \mathrm{H}, \mathrm{CH}_{2}$ ), $1.68\left(\mathrm{~d}, J=7 \mathrm{~Hz}, 3 \mathrm{H}, \mathrm{CH}_{3} \mathrm{CH}\right), 1.34(\mathrm{t}, J=7.5 \mathrm{~Hz}, 3 \mathrm{H}$, $\left.\mathrm{CH}_{3} \mathrm{CH}_{2}\right)$.

${ }^{13} \mathrm{CNMR}\left(\mathrm{CDCl}_{3}\right): \delta=172.6(\mathrm{~s}, \mathrm{C}=\mathrm{O}), 159.9(\mathrm{~s}, \mathrm{C} 4)$, 158.4 (s, C2), 156.8 (s, C1 of OPh), 147.0 (s, C8a), 134.8 (d, C7), 129.9 (d, C3 of OPh), 127.5 (d, C5), 126.9 (d, C6), 126.7 (d, C8), 122.9 (d, C4 of OPh), 120.7 (s, $\mathrm{C} 4 \mathrm{a}), 116.5$ (d, C2 of OPh), $75.8(\mathrm{~d}, \mathrm{CH}), 26.8\left(\mathrm{t}, \mathrm{CH}_{2}\right)$, 18.7 (q, $\left.\mathrm{CH}_{3} \mathrm{CH}\right), 10.6$ (q, $\mathrm{CH}_{3} \mathrm{CH}_{2}$ ).

\section{2-Ethyl-3-[2-(1-}

\section{naphthalene)propionylamino]quinazolin-4(3H)-one}

(6)

mp $180-182{ }^{\circ} \mathrm{C} ; \mathbf{6 a} / \mathbf{6 b}=5: 6\left({ }^{13} \mathrm{C} \mathrm{NMR}\right)$.

EI-MS: $m / z(\%)=371\left(\mathrm{M}^{+}, 5\right), 216(40), 182(38), 155$ (100), 153 (34), 130 (15), 115 (9), 90 (8), 76 (10).

CI-MS: $m / z(\%)=372\left(\mathrm{MH}^{+}, 7\right), 217$ (9), $175(100), 159$ (7), 91 (6).

HRMS: $m / z$ calcd for $\mathrm{C}_{23} \mathrm{H}_{21} \mathrm{~N}_{3} \mathrm{O}_{2}\left(\mathrm{MH}^{+}\right)$: 371.1634; found: 371.1634 .

${ }^{1} \mathrm{H}$ NMR $\left(\mathrm{CDCl}_{3}\right): \delta=8.20-7.37(\mathrm{~m}, 12 \mathrm{H}, \mathrm{ArH}$ and $\mathrm{NH})$, 4.71-4.62 (m, $1 \mathrm{H}, \mathrm{CH}), 2.54-2.28\left(\mathrm{~m}, 2 \mathrm{H}, \mathrm{CH}_{2}\right)$, 
1.89, $1.74\left(2 \mathrm{~d}, J=7 \mathrm{~Hz}, 3 \mathrm{H}, \mathrm{CH}_{3} \mathrm{CH}\right), 0.99(\mathrm{t}, J=7.5$ $\mathrm{Hz}, 3 \mathrm{H}, \mathrm{CH}_{3} \mathrm{CH}_{2}$ ).

\section{Compound 6a}

${ }^{13} \mathrm{C}$ NMR $\left(\mathrm{CDCl}_{3}\right): \delta=175.0(\mathrm{~s}, \mathrm{C}=\mathrm{O}), 160.2(\mathrm{~s}, \mathrm{C} 4)$, 158.8 (s, C2), 146.9 (s, C8a), 136.0 (s, C1'), 134.8 (d, C7), 133.8 (s, C4'a), 131.1 (s, C8'a), 129.1 (d, C5'), 128.7 (d, C5), 127.8 (d, C6), 126.8 (d, C8), 126.7 (d, C3'), 126.5 (d, C2'), 126.1 (d, C4'), 125.8 (d, C7'), 124.9 (d, C6'), $123.2\left(\mathrm{~d}, \mathrm{C} 8^{\prime}\right), 120.6$ (s, C4a), 42.0 (d, CH), 26.8 (t, $\mathrm{CH}_{2}$ ), 17.7 (q, $\left.\mathrm{CH}_{3} \mathrm{CH}\right), 10.5$ (q, $\mathrm{CH}_{3} \mathrm{CH}_{2}$ ).

\section{Compound 6b}

${ }^{13} \mathrm{C} \mathrm{NMR}\left(\mathrm{CDCl}_{3}\right): \delta=174.9(\mathrm{~s}, \mathrm{C}=\mathrm{O}), 160.0(\mathrm{~s}, \mathrm{C} 4)$, 158.7 (s, C2), 146.9 (s, C8a), 136.0 (s, C1'), 134.7 (d, C7), 134.2 (s, C4'a), 131.1 (s, C8'a), 129.2 (d, C5'), 128.5 (d, C5), 127.3 (d, C6), 126.8 (d, C8), 126.7 (d, $\mathrm{C}^{\prime}$ ), 126.5 (d, C2'), 126.2 (d, C4'), 125.9 (d, C7'), 125.6 (d, C6'), 122.8 (d, C8'), 120.5 (s, C4a), 42.0 (d, CH), $26.9\left(\mathrm{t}, \mathrm{CH}_{2}\right), 17.6$ (q, $\left.\mathrm{CH}_{3} \mathrm{CH}\right), 10.5\left(\mathrm{q}, \mathrm{CH}_{3} \mathrm{CH}_{2}\right)$.

\section{2-Ethyl-3-(2-phenoxybutyrylamino)quinazolin-4(3H)- one (7)}

mp 93-94 ${ }^{\circ} \mathrm{C} ; \mathbf{7 a} / \mathbf{7 b}=1: 2\left({ }^{1} \mathrm{H}\right.$ NMR).

Anal. Calcd for $\mathrm{C}_{20} \mathrm{H}_{21} \mathrm{~N}_{3} \mathrm{O}_{3}: \mathrm{C}, 68.36 ; \mathrm{H}, 6.02 ; \mathrm{N}, 11.96$. Found: C, 68.4; H, 6.2; N, 11.8 .

EI-MS: $m / z(\%)=351\left(\mathrm{M}^{+}, 10\right), 258(7), 230(13), 216$ (60), 173 (40), 135 (100), 107 (65), 94 (31), 77 (75), 41 (43).

CI-MS: $m / z(\%)=352\left(\mathrm{MH}^{+}, 10\right), 197(25), 176(18)$, 175 (100), 136 (5), 105 (7), 58 (5).

HRMS: $m / z$ calcd for $\mathrm{C}_{20} \mathrm{H}_{21} \mathrm{~N}_{3} \mathrm{O}_{3}\left(\mathrm{M}^{+}\right)$: 351.1583; found: 351.1583 .

\section{Compound 7a}

${ }^{1} \mathrm{H}$ NMR $\left(\mathrm{CDCl}_{3}\right): \delta=8.69$ (s, exch., $\left.1 \mathrm{H}, \mathrm{NH}\right), 8.16(\mathrm{~d}$, $J=8 \mathrm{~Hz}, 1 \mathrm{H}, \mathrm{H} 5$ ), 7.73 (app. dt, $J=8,1 \mathrm{~Hz}, 1 \mathrm{H}, \mathrm{H} 7$ ), $7.60(\mathrm{~d}, J=8 \mathrm{~Hz}, 1 \mathrm{H}, \mathrm{H} 8), 7.40(\mathrm{t}, J=8 \mathrm{~Hz}, 1 \mathrm{H}, \mathrm{H} 6)$, $7.36(\mathrm{t}, J=7.5 \mathrm{~Hz}, 2 \mathrm{H}, \mathrm{H} 3 \mathrm{of} \mathrm{OPh}), 7.18-6.99(\mathrm{~m}, 3 \mathrm{H}$, $\mathrm{H} 2$ and $\mathrm{H} 4$ of $\mathrm{OPh}), 4.78(\mathrm{t}, J=7 \mathrm{~Hz}, 1 \mathrm{H}, \mathrm{CH}), 2.41$ (dq, $J=14,7 \mathrm{~Hz}, 2 \mathrm{H}, \mathrm{CH}_{2} \mathrm{CH}$ ), 2.18 (dq, $J=15,7.5 \mathrm{~Hz}$, $\left.2 \mathrm{H}, \mathrm{CH}_{2}\right), 1.20$ (t, $\left.J=7 \mathrm{~Hz}, 3 \mathrm{H}, \mathrm{CH}_{3} \mathrm{CH}_{2} \mathrm{CH}\right), 1.02$ (t, $J$ $=7.5 \mathrm{~Hz}, 3 \mathrm{H}, \mathrm{CH}_{3} \mathrm{CH}_{2}$ ).

${ }^{13} \mathrm{C} \mathrm{NMR}\left(\mathrm{CDCl}_{3}\right): \delta=172.1(\mathrm{~s}, \mathrm{C}=\mathrm{O}), 159.9(\mathrm{~s}, \mathrm{C} 4)$, 158.5 (s, C2), $157.2(\mathrm{~s}, \mathrm{C} 1 \mathrm{of} \mathrm{OPh}), 146.9$ (s, C8a), 134.8 (d, C7), 129.9 (d, C3 of OPh), 127.0 (d, C5), 126.6 (d, C6), 126.5 (d, C8), 122.4 (d, C4 of OPh), 120.5 (s, $\mathrm{C} 4 \mathrm{a}), 115.1(\mathrm{~d}, \mathrm{C} 2$ of $\mathrm{OPh}), 79.5(\mathrm{~d}, \mathrm{CH}), 26.9(\mathrm{t}$, $\mathrm{CH}_{2} \mathrm{CH}$ ), 26.5 (t, $\mathrm{CH}_{2}$ ), 10.5 (q, $\left.\mathrm{CH}_{3} \mathrm{CH}_{2} \mathrm{CH}\right), 9.5$ (q, $\mathrm{CH}_{3} \mathrm{CH}_{2}$ ).

\section{Compound 7b}

${ }^{1} \mathrm{H} \mathrm{NMR}\left(\mathrm{CDCl}_{3}\right): \delta=8.88$ (s, exch., $\left.1 \mathrm{H}, \mathrm{NH}\right), 8.16(\mathrm{~d}$, $J=8 \mathrm{~Hz}, 1 \mathrm{H}, \mathrm{H} 5$ ), 7.43 (app. dt, $J=8,1 \mathrm{~Hz}, 1 \mathrm{H}, \mathrm{H} 7$ ), $7.60(\mathrm{~d}, J=8 \mathrm{~Hz}, 1 \mathrm{H}, \mathrm{H} 8), 7.40(\mathrm{t}, J=8 \mathrm{~Hz}, 1 \mathrm{H}, \mathrm{H} 6)$, 7.36 (t, $J=7.5 \mathrm{~Hz}, 2 \mathrm{H}, \mathrm{H} 3 \mathrm{of} \mathrm{OPh}), 7.18-6.99(\mathrm{~m}, 3 \mathrm{H}$,
$\mathrm{H} 2$ and $\mathrm{H} 4$ of $\mathrm{OPh}), 4.78(\mathrm{t}, J=7.4 \mathrm{~Hz}, 1 \mathrm{H}, \mathrm{CH}), 2.87$ (dq, $J=14,7 \mathrm{~Hz}, 2 \mathrm{H}, \mathrm{CH}_{2} \mathrm{CH}$ ), 2.08 (dq, $J=15,7.5 \mathrm{~Hz}$, $\left.2 \mathrm{H}, \mathrm{CH}_{2}\right), 1.33$ (t, $\left.3 \mathrm{H}, J=7 \mathrm{~Hz}, \mathrm{CH}_{3} \mathrm{CH}_{2} \mathrm{CH}\right), 1.11$ (t, $J$ $=7.5 \mathrm{~Hz}, 3 \mathrm{H}, \mathrm{CH}_{3} \mathrm{CH}_{2}$ ).

${ }^{13} \mathrm{C}$ NMR $\left(\mathrm{CDCl}_{3}\right): \delta=172.1(\mathrm{~s}, \mathrm{C}=\mathrm{O}), 159.8(\mathrm{~s}, \mathrm{C} 4)$, 158.3 (s, C2), 157.5 (s, C1 of OPh), 146. 9 (s, C8a), 134.8 (d, C7), 127.4 (d, C3 of OPh), 127.0 (d, C5), 126.8 (d, C6), 126.7 (d, C8), 122.9 (d, C4 of OPh), 120.6 (s, C4a), 116.7 (d, C2 of $\mathrm{OPh}), 80.9(\mathrm{~d}, \mathrm{CH}), 26.9(\mathrm{t}$, $\mathrm{CH}_{2} \mathrm{CH}$ ), 26.1 (t, $\mathrm{CH}_{2}$ ), 10.3 (q, $\mathrm{CH}_{3} \mathrm{CH}_{2} \mathrm{CH}$ ), 9.2 (q, $\mathrm{CH}_{3} \mathrm{CH}_{2}$ ).

\section{2-Ethyl-3-(2-phenylbutyrylamino)quinazolin-4(3H)- one (8)}

mp $180-183{ }^{\circ} \mathrm{C} ; \mathbf{8 a} / \mathbf{8 b}=1: 3\left({ }^{1} \mathrm{H}\right.$ NMR $)$.

EI-MS: $m / z(\%)=335\left(\mathrm{M}^{+}, 5\right), 216(40), 189(17), 173$ (12), 119 (65), 91 (100).

HRMS: $m / z$ calcd for $\mathrm{C}_{20} \mathrm{H}_{21} \mathrm{~N}_{3} \mathrm{O}_{2}\left(\mathrm{M}^{+}\right)$: 335.1634; found: 335.1634 .

\section{Compound 8a}

${ }^{1} \mathrm{H}$ NMR (DMSO- $d_{6}$ ): $\delta=11.66$ (s, exch., $1 \mathrm{H}, \mathrm{NH}$ ), 8.10 (d, $J=8 \mathrm{~Hz}, 1 \mathrm{H}, \mathrm{H} 5), 7.99$ (t, $J=8 \mathrm{~Hz}, 1 \mathrm{H}, \mathrm{H} 7)$, $7.75(\mathrm{t}, J=8 \mathrm{~Hz}, 1 \mathrm{H}, \mathrm{H} 6), 7.52-7.30(\mathrm{~m}, 6 \mathrm{H}, \mathrm{H} 8$ and $\mathrm{Ph}), 3.98(\mathrm{t}, J=7 \mathrm{~Hz}, 1 \mathrm{H}, \mathrm{CH}), 3.00,3.62(2 \mathrm{dq}, J=14$, $\left.7 \mathrm{~Hz}, 2 \mathrm{H}, \mathrm{CH}_{2} \mathrm{CH}\right), 2.22,1.91(2 \mathrm{dq}, J=15,7.5 \mathrm{~Hz}, 2$ $\left.\mathrm{H}, \mathrm{CH}_{2}\right), 1.05$ (t, $\left.J=7 \mathrm{~Hz}, 3 \mathrm{H}, \mathrm{CH}_{3} \mathrm{CH}_{2} \mathrm{CH}\right), 1.00$ (t, $J=$ $7.5 \mathrm{~Hz}, 3 \mathrm{H}, \mathrm{CH}_{3} \mathrm{CH}_{2}$ ).

${ }^{13} \mathrm{C}$ NMR (DMSO- $\left.d_{6}\right): \delta=173.7(\mathrm{~s}, \mathrm{C}=\mathrm{O}), 167.8(\mathrm{~s}, \mathrm{C} 4)$, 155.9 (s, C2), 138.7 (s, C8a), 138.0 (s, C1 of Ph), 136.7 (d, C7), 129.5 (d, C5), 128.8 (d, C3 of Ph), 128.3 (d, C6), 128.0 (d, C2 of Ph), 127.6 (d, C8), 120.1 (d, C4 of $\mathrm{Ph}), 118.5$ (s, C4a), $52.3(\mathrm{~d}, \mathrm{CH}), 26.6\left(\mathrm{t}, \mathrm{CH}_{2} \mathrm{CH}\right), 25.4$ (t, $\mathrm{CH}_{2}$ ), 12.1 (q, $\mathrm{CH}_{3} \mathrm{CH}_{2} \mathrm{CH}$ ), 11.3 (q, $\mathrm{CH}_{3} \mathrm{CH}_{2}$ ).

\section{Compound 8b}

${ }^{1} \mathrm{H}$ NMR (DMSO- $d_{6}$ ): $\delta=11.38$ (s, exch., $1 \mathrm{H}, \mathrm{NH}$ ), $8.10(\mathrm{~d}, J=8 \mathrm{~Hz}, 1 \mathrm{H}, \mathrm{H} 5), 7.99$ (t, $J=8 \mathrm{~Hz}, 1 \mathrm{H} \mathrm{H} 7)$, $7.75(\mathrm{t}, J=8 \mathrm{~Hz}, 1 \mathrm{H}, \mathrm{H} 6), 7.52-7.30(\mathrm{~m}, 6 \mathrm{H}, \mathrm{H} 8$ and $\mathrm{Ph}), 3.90(\mathrm{t}, J=7 \mathrm{~Hz}, 1 \mathrm{H}, \mathrm{CH}), 3.34,3.10(2 \mathrm{dq}, J=14$, $\left.7 \mathrm{~Hz}, 2 \mathrm{H}, \mathrm{CH}_{2} \mathrm{CH}\right), 2.22,1.91(2 \mathrm{dq}, J=15,7.5 \mathrm{~Hz}, 2$ $\left.\mathrm{H}, \mathrm{CH}_{2}\right), 1.44\left(\mathrm{t}, J=7 \mathrm{~Hz}, 3 \mathrm{H}, \mathrm{CH}_{3} \mathrm{CH}_{2} \mathrm{CH}\right), 1.03$ (t, $J=$ $\left.7.5 \mathrm{~Hz}, 3 \mathrm{H}, \mathrm{CH}_{3} \mathrm{CH}_{2}\right)$.

${ }^{13} \mathrm{C}$ NMR (DMSO- $\left.d_{6}\right): \delta=173.8(\mathrm{~s}, \mathrm{C}=\mathrm{O}), 167.6(\mathrm{~s}, \mathrm{C} 4)$, 155.7 (s, C2), 138.7 (s, C8a), 138.0 (s, C1 of Ph), 136.4 (d, C7), 129.6 (d, C5), 128.6 (d, C3 of Ph), 128.3 (d, C6), 128.2 (d, C2 of Ph), 127.6 (d, C8), 120.2 (d, C4 of $\mathrm{Ph}), 118.5$ (s, C4a), 52.7 (d, CH), $26.60\left(\mathrm{t}, \mathrm{CH}_{2} \mathrm{CH}\right), 25.1$ (t, $\left.\mathrm{CH}_{2}\right), 12.2$ (q, $\left.\mathrm{CH}_{3} \mathrm{CH}_{2} \mathrm{CH}\right), 11.9$ (q, $\mathrm{CH}_{3} \mathrm{CH}_{2}$ ).

\section{2-Propyl-3-(2-phenoxypropionylamino)quinazolin- 4(3H)-one (9)}

mp 87-89 ${ }^{\circ} \mathrm{C} ; \mathbf{9 a} / \mathbf{9 b}=1: 2\left({ }^{1} \mathrm{H}\right.$ NMR $)$.

EI-MS: $m / z(\%)=352\left(\mathrm{M}^{+}+1,12\right), 337\left(\mathrm{M}^{+}, 20\right), 323$ (100), 285 (35). 
CI-MS: $m / z(\%)=352\left(\mathrm{MH}^{+}, 16\right), 189(100), 183(32)$, 91 (17).

HRMS: $m / z$ calcd for $\mathrm{C}_{20} \mathrm{H}_{22} \mathrm{~N}_{3} \mathrm{O}_{3}\left(\mathrm{MH}^{+}\right)$: 352.1661 ; found: 352.1664 .

\section{Compound 9a}

${ }^{1} \mathrm{H}$ NMR $\left(\mathrm{CDCl}_{3}\right): \delta=8.98$ (s, exch., $\left.1 \mathrm{H}, \mathrm{NH}\right), 8.17$ (dd, $J=8,1 \mathrm{~Hz}, 1 \mathrm{H}, \mathrm{H} 5), 7.76-7.60$ (m, $2 \mathrm{H}, \mathrm{H} 7$ and $\mathrm{H} 8)$, 7.42 (app. dt, $J=8,1 \mathrm{~Hz}, 1 \mathrm{H}, \mathrm{H} 6), 7.37-7.33$ (m, $1 \mathrm{H}$, $\mathrm{H} 3$ of OPh), 7.10-7.06 (m, $1 \mathrm{H}, \mathrm{H} 4$ of OPh), 7.00 (d, $J=$ $7.5 \mathrm{~Hz}, 1 \mathrm{H}, \mathrm{H} 2$ of OPh), $4.96(\mathrm{q}, J=7 \mathrm{~Hz}, 1 \mathrm{H}, \mathrm{CH})$, 2.79-2.73 (m, $\left.2 \mathrm{H}, \mathrm{CH}_{2}\right), 1.79(\mathrm{~d}, J=7 \mathrm{~Hz}, 3 \mathrm{H}$, $\left.\mathrm{CHCH}_{3}\right), 1.63-1.49\left(\mathrm{~m}, 2 \mathrm{H}, \mathrm{CH}_{2}\right), 1.05(\mathrm{t}, J=7.5 \mathrm{~Hz}, 3$ $\mathrm{H}, \mathrm{CH}_{3} \mathrm{CH}_{2}$ ).

${ }^{13} \mathrm{C} \mathrm{NMR}\left(\mathrm{CDCl}_{3}\right): \delta=172.4(\mathrm{~s}, \mathrm{C}=\mathrm{O}), 159.9$ (s, C4), 157.5 (s, C1 of OPh), 156.8 (s, C2), 146.9 (s, C8a), 134.9 (d, C7), 129.9 (d, C3 of OPh), 126.9 (d, C5), 126.8 (d, C6), 126.6 (d, C8), 122.8 (d, C4 of OPh), 120.7 (s, C4a), 116.5 (d, C2 of OPh), 75.7 (d, CH), $35.6\left(\mathrm{t}, \mathrm{CH}_{2}\right)$, 20.0 (t, $\mathrm{CH}_{2}$ ), 18.7 (q, $\left.\mathrm{CH}_{3} \mathrm{CH}\right), 13.9$ (q, $\mathrm{CH}_{3} \mathrm{CH}_{2}$ ).

\section{Compound 9b}

${ }^{1} \mathrm{H}$ NMR $\left(\mathrm{CDCl}_{3}\right): \delta=8.75$ (s, exch., $\left.1 \mathrm{H}, \mathrm{NH}\right), 8.17(\mathrm{dd}$, $J=8,1 \mathrm{~Hz}, 1 \mathrm{H}, \mathrm{H} 5), 7.76-7.60$ (m, $2 \mathrm{H}, \mathrm{H} 7$ and H8), 7.42 (app. dt, $J=8,1 \mathrm{~Hz}, 1 \mathrm{H}, \mathrm{H} 6), 7.37-7.33$ (m, $1 \mathrm{H}$, $\mathrm{H} 3$ of OPh), 7.10-7.06 (m, $1 \mathrm{H}, \mathrm{H} 4$ of OPh), $7.00(\mathrm{~d}, J=$ $7.5 \mathrm{~Hz}, 1 \mathrm{H}, \mathrm{H} 2$ of OPh), $4.94(\mathrm{q}, J=7 \mathrm{~Hz}, 1 \mathrm{H}, \mathrm{CH})$, 2.44-2.38 (m, $\left.2 \mathrm{H}, \mathrm{CH}_{2}\right), 1.83(\mathrm{~d}, J=7 \mathrm{~Hz}, 3 \mathrm{H}$, $\left.\mathrm{CH}_{3} \mathrm{CH}\right), 1.63-1.49\left(\mathrm{~m}, 2 \mathrm{H}, \mathrm{CH}_{2}\right), 0.80(\mathrm{t}, J=7.4 \mathrm{~Hz}, 3$ $\mathrm{H}, \mathrm{CH}_{3} \mathrm{CH}_{2}$ ).

${ }^{13} \mathrm{C} \mathrm{NMR}\left(\mathrm{CDCl}_{3}\right): \delta=172.9(\mathrm{~s}, \mathrm{C}=\mathrm{O}), 159.9$ (s, C4), 157.7 (s, C1 of OPh), 156.9 (s, C2), 146.9 (s, C8a), 134.9 (d, C7), 130.0 (d, C3 of OPh), 127.4 (d, C5), 126.8 (d, C6), 126.6 (d, C8), 122.5 (d, C4 of OPh), 120.5 (s, $\mathrm{C} 4 \mathrm{a}), 116.5$ (d, C2 of OPh), $74.6(\mathrm{~d}, \mathrm{CH}), 35.5\left(\mathrm{t}, \mathrm{CH}_{2}\right)$, 19.9 (t, $\mathrm{CH}_{2}$ ), 18.9 (q, $\left.\mathrm{CH}_{3} \mathrm{CH}\right), 13.6$ (q, $\mathrm{CH}_{3} \mathrm{CH}_{2}$ ).

\section{2-Propyl-3-(2-phenoxybutyrylamino)quinazolin- 4(3H)-one (10)}

mp $115-117^{\circ} \mathrm{C} ; \mathbf{1 0 a} / \mathbf{1 0 b}=1: 2\left({ }^{1} \mathrm{H}\right.$ NMR $)$.

EI-MS: $m / z(\%)=365\left(\mathrm{M}^{+}, 4\right), 337$ (6), 244 (15), 230 (30), 187 (20), 173 (20), 160 (60), 135 (45), 117 (34), 107 (40), 94 (43), 77 (100), 65 (35).

CI-MS: $m / z(\%)=366\left(\mathrm{MH}^{+}, 30\right), 197$ (32), 189 (100), 173 (13), 136 (5), 105 (33).

HRMS: $m / z$ calcd for $\mathrm{C}_{21} \mathrm{H}_{24} \mathrm{~N}_{3} \mathrm{O}_{3}\left(\mathrm{MH}^{+}\right)$: 366.1819 ; found: 366.1819 .

\section{Compound 10a}

${ }^{1} \mathrm{H}$ NMR $\left(\mathrm{CDCl}_{3}\right): \delta=8.39$ (s, exch., $\left.1 \mathrm{H}, \mathrm{NH}\right), 8.16(\mathrm{~m}$, $1 \mathrm{H}, \mathrm{H} 5), 7.71$ (m, $1 \mathrm{H}, \mathrm{H} 7), 7.60$ (d, J=8 Hz, $1 \mathrm{H}, \mathrm{H} 8)$, 7.44-7.00 (m, $6 \mathrm{H}, \mathrm{H} 6$ and $\mathrm{OPh}), 4.81-4.77(\mathrm{~m}, 1 \mathrm{H}$, $\mathrm{CH})$, 2.74-2.70 (m, $\left.2 \mathrm{H}, \mathrm{CH}_{2}\right), 2.13-2.81\left(\mathrm{~m}, 2 \mathrm{H}, \mathrm{CH}_{2}\right)$, 1.84-1.79 (m, $\left.2 \mathrm{H}, \mathrm{CH}_{2}\right), 1.12\left(\mathrm{t}, J=7 \mathrm{~Hz}, 3 \mathrm{H}, \mathrm{CH}_{3}\right)$, $1.04\left(\mathrm{t}, J=7.5 \mathrm{~Hz}, 3 \mathrm{H}, \mathrm{CH}_{3}\right)$.
${ }^{13} \mathrm{C}$ NMR $\left(\mathrm{CDCl}_{3}\right): \delta=172.1(\mathrm{~s}, \mathrm{C}=\mathrm{O}), 159.8(\mathrm{~s}, \mathrm{C} 4)$, 157.5 (s, C1 of OPh), 157.5 (s, C2), 146.9 (s, C8a), 134.8 (d, C7), 129.8 (d, C3 of OPh), 127.4 (d, C5), 126.8 (d, C6), 126.6 (d, C8), 122.4 (d, C4 of OPh), 120.7 (s, $\mathrm{C} 4 \mathrm{a}), 116.7$ (d, C2 of $\mathrm{OPh}), 80.9(\mathrm{~d}, \mathrm{CH}), 35.7\left(\mathrm{t}, \mathrm{CH}_{2}\right)$, $26.1\left(\mathrm{t} \mathrm{CH}_{2}\right), 20.0$ (t, $\left.\mathrm{CH}_{2}\right), 13.8$ (q, $\left.\mathrm{CH}_{3}\right), 9.1$ (q, $\left.\mathrm{CH}_{3}\right)$.

\section{Compound $10 b$}

${ }^{1} \mathrm{H}$ NMR $\left(\mathrm{CDCl}_{3}\right): \delta=8.73$ (s, exch., $\left.1 \mathrm{H}, \mathrm{NH}\right), 8.16(\mathrm{~m}$, $1 \mathrm{H}, \mathrm{H} 5), 7.71$ (m, $1 \mathrm{H}, \mathrm{H} 7), 7.60$ (d, $J=8 \mathrm{~Hz}, 1 \mathrm{H}, \mathrm{H} 8)$, 7.44-7.00 (m, $6 \mathrm{H}, \mathrm{H} 6$ and $\mathrm{OPh}), 4.81-4.77(\mathrm{~m}, 1 \mathrm{H}$, $\mathrm{CH}), 2.35-2.31\left(\mathrm{~m}, 2 \mathrm{H}, \mathrm{CH}_{2}\right), 2.25-2.20\left(\mathrm{~m}, 2 \mathrm{H}, \mathrm{CH}_{2}\right)$, 1.57-1.52 (m, $\left.2 \mathrm{H}, \mathrm{CH}_{2}\right), 1.21\left(\mathrm{t}, J=7 \mathrm{~Hz}, 3 \mathrm{H}, \mathrm{CH}_{3}\right)$, $0.76\left(\mathrm{t}, J=7.5 \mathrm{~Hz}, 3 \mathrm{H}, \mathrm{CH}_{3}\right)$.

${ }^{13} \mathrm{C} \mathrm{NMR}\left(\mathrm{CDCl}_{3}\right): \delta=172.2(\mathrm{~s}, \mathrm{C}=\mathrm{O}), 159.9(\mathrm{~s}, \mathrm{C} 4)$, 157.7 (s, C1 of OPh), 157.3 (s, C2), 146.9 (s, C8a), 134.8 (d, C7), 129.9 (d, C3 of OPh), 127.0 (d, C5), 126.8 (d, C6), 126.6 (d, C8), 122.9 (d, C4 of OPh), 120.5 (s, $\mathrm{C} 4 \mathrm{a}), 115.0$ (d, C2 of OPh), $79.8(\mathrm{~d}, \mathrm{CH}), 35.5\left(\mathrm{t}, \mathrm{CH}_{2}\right)$, $26.6\left(\mathrm{t} \mathrm{CH}_{2}\right), 19.8\left(\mathrm{t}, \mathrm{CH}_{2}\right), 13.6\left(\mathrm{q}, \mathrm{CH}_{3}\right), 9.6\left(\mathrm{q}, \mathrm{CH}_{3}\right)$.

\section{2-Substituted 3-Acylaminoquinazolin-4(3H)-ones (13- 27); General Procedure}

A solution of LDA in pentane (1.6 M; $1.3 \mathrm{~mL}, 2.2 \mathrm{mmol})$ was added under $\mathrm{N}_{2}$ in a dropwise manner to a stirred solution of the appropriate 3-acylamino-2alkylquinazolin-4(3H)-ones (5-10, $1 \mathrm{mmol})$ in anhydrous THF $(10 \mathrm{~mL})$ at $-78{ }^{\circ} \mathrm{C}$. Formation of the dilithio reagent was observed as a very deep red solution. The mixture was stirred at $-78^{\circ} \mathrm{C}$ for $30 \mathrm{~min}$, after which an electrophile $(1.1 \mathrm{mmol})$ (as a solution in anhydrous THF for benzophenone, otherwise neat) was added. The mixture was stirred for $2 \mathrm{~h}$ at $-78^{\circ} \mathrm{C}$ then removed from the cooling bath and allowed to warm to r.t., diluted with $\mathrm{Et}_{2} \mathrm{O}(10 \mathrm{~mL})$, and quenched with sat. aq. $\mathrm{NH}_{4} \mathrm{Cl}$ solution $(10 \mathrm{~mL})$. The organic layer was washed with $\mathrm{H}_{2} \mathrm{O}$ $(2 \times 20 \mathrm{~mL})$, dried $\left(\mathrm{MgSO}_{4}\right)$, and evaporated under reduced pressure. The products obtained were purified by column chromatography on silica gel $\left(\mathrm{Et}_{2} \mathrm{O}\right.$-hexane, 1:4) to give the corresponding 2-substituted derivatives 13-27 as white powders. The yields obtained are recorded in Table 2 .

\section{$\left(R_{\mathrm{a}}{ }^{*}\right)-2-\left(\left(1 S^{*}\right)-2,2-D i p h e n y l-2-h y d r o x y-1-\right.$ methylethyl)-3-((2R*)-2-} phenoxypropionylamino)quinazolin-4(3H)-one (13a)

mp $172^{\circ} \mathrm{C}$; a single diastereoisomer (NMR).

Anal. Calcd for $\mathrm{C}_{32} \mathrm{H}_{29} \mathrm{~N}_{3} \mathrm{O}_{4}$. THF: C, 73.08; H, 6.30; N, 7.10. Found: C, 73.2; H, 6.2; N, 7.3.

EI-MS: $m / z(\%)=355$ (5), 337 (10), 216 (42), 182 (49), 173 (37), 121 (50), 105 (100), 77 (95), 51 (42).

CI-MS: $m / z(\%)=520\left(\mathrm{MH}^{+}, 33\right), 357$ (100), 338 (40), 262 (20), 228 (15).

HRMS: $m / z$ calcd for $\mathrm{C}_{32} \mathrm{H}_{30} \mathrm{~N}_{3} \mathrm{O}_{4}\left(\mathrm{MH}^{+}\right)$: 520.2236; found: 520.2206 . 
${ }^{1} \mathrm{H}$ NMR $\left(\mathrm{CDCl}_{3}\right): \delta=8.34$ (s, exch., $\left.1 \mathrm{H}, \mathrm{NH}\right), 8.09(\mathrm{dd}$, $J=8,1 \mathrm{~Hz}, 1 \mathrm{H}, \mathrm{H} 5$ ), 7.73 (app. dt, $J=8,1 \mathrm{~Hz}, 1 \mathrm{H}$, H7), $7.61(\mathrm{~d}, J=8 \mathrm{~Hz}, 1 \mathrm{H}, \mathrm{H} 8), 7.51-6.92(\mathrm{~m}, 17 \mathrm{H}$, ArH and $\mathrm{OH}), 5.02(\mathrm{q}, J=6.7 \mathrm{~Hz}, 1 \mathrm{H}, \mathrm{CH}), 4.00(\mathrm{q}, J=$ $7 \mathrm{~Hz}, 1 \mathrm{H}, \mathrm{CH}), 3.75(\mathrm{t}, J=6.5 \mathrm{~Hz}, 4 \mathrm{H}, \mathrm{H} 2$ of THF), $1.86(\mathrm{t}, J=6.5 \mathrm{~Hz}, 4 \mathrm{H}, \mathrm{H} 3$ of THF $), 1.82(\mathrm{~d}, J=6.7 \mathrm{~Hz}$, $\left.3 \mathrm{H}, \mathrm{CH}_{3}\right), 1.30\left(\mathrm{~d}, J=7 \mathrm{~Hz}, 3 \mathrm{H}, \mathrm{CH}_{3}\right)$.

${ }^{13} \mathrm{C} \mathrm{NMR}\left(\mathrm{CDCl}_{3}\right): \delta=173.7(\mathrm{~s}, \mathrm{C}=\mathrm{O}), 161.6(\mathrm{~s}, \mathrm{C} 4)$, $159.0(\mathrm{~s}, \mathrm{C} 2), 157.2(\mathrm{~s}, \mathrm{C} 1$ of $\mathrm{OPh}), 147.6(\mathrm{~s}, \mathrm{C} 8 \mathrm{a})$, $145.3,144.3(2 \mathrm{~s}, \mathrm{C} 1$ of $2 \mathrm{Ph}), 135.2(\mathrm{~d}, \mathrm{C} 7), 130.4(\mathrm{~d}$, $\mathrm{C} 3$ of $\mathrm{OPh}), 128.1$ (d, C5), $127.2127 .0(2 \mathrm{~d}, \mathrm{C} 3$ of $2 \mathrm{Ph})$, 126.9 (d, C6), 126.6 (d, C8), 126.5, 125.5 (2 d, C2 of 2 $\mathrm{Ph}), 124.7$ (d, C4 of $2 \mathrm{Ph}), 122.7(\mathrm{~d}, \mathrm{C} 4$ of OPh), 120.2 (s, C4a), 114.4 (d, C2 of OPh), 79.2 (s, C-OH), 74.5 (d, $\mathrm{CH}), 68.0(\mathrm{t}, \mathrm{C} 2$ of THF), $42.1(\mathrm{~d}, \mathrm{CH}), 25.6(\mathrm{t}, \mathrm{C} 3$ of THF), 19.2 (q, $\left.\mathrm{CH}_{3}\right), 15.1$ (q, $\mathrm{CH}_{3}$ ).

\section{2-(2,2-Diphenyl-2-hydroxy-1-methylethyl)-3-(2-} phenoxypropionylamino)quinazolin- $4(3 H)$-one (13)

mp $171-173^{\circ} \mathrm{C}$; four diastereoisomers (NMR).

Anal. Calcd for $\mathrm{C}_{32} \mathrm{H}_{29} \mathrm{~N}_{3} \mathrm{O}_{4}$ : C, 73.97; H, 5.63; N, 8.09. Found: C, 74.2; H, 5.6; N, 8.0.

EI-MS: $m / z(\%)=355(6), 337(12), 216(42), 182(30)$, 173 (20), 121 (55), 105 (90), 77 (100), 51 (41).

CI-MS: $m / z(\%)=520\left(\mathrm{MH}^{+}, 45\right), 357$ (90), $338(100)$.

HRMS: $m / z$ calcd for $\mathrm{C}_{32} \mathrm{H}_{30} \mathrm{~N}_{3} \mathrm{O}_{4}\left(\mathrm{MH}^{+}\right)$: 520.2236; found: 520.2198 .

${ }^{1} \mathrm{H}$ NMR $\left(\mathrm{CDCl}_{3}\right): \delta=8.58,8.55(2 \mathrm{~s}$, exch., $1 \mathrm{H}, \mathrm{NH})$, 8.09, 7.85 ( $2 \mathrm{dd}, J=8,1 \mathrm{~Hz}, 1 \mathrm{H}, \mathrm{H} 5), 7.80-6.85$ (m, 19 $\mathrm{H}, \operatorname{ArH}$ and $\mathrm{OH}), 5.12,5.02(2 \mathrm{q}, J=7 \mathrm{~Hz}, 1 \mathrm{H}, \mathrm{CH})$, 4.36, 4.01, 3.96, $3.73(4 \mathrm{q}, J=7 \mathrm{~Hz}, 1 \mathrm{H}, \mathrm{CH}), 1.94$, $1.86,1.76\left(3 \mathrm{~d}, J=7 \mathrm{~Hz}, 3 \mathrm{H}, \mathrm{CH}_{3}\right), 1.36,1.30,0.75$ (3 d, $\left.J=7 \mathrm{~Hz}, 3 \mathrm{H}, \mathrm{CH}_{3}\right)$.

${ }^{13} \mathrm{C}$ NMR $\left(\mathrm{CDCl}_{3}\right): \delta=173.6,173.4(2 \mathrm{~s}, \mathrm{C}=\mathrm{O}), 161.5$ (s, C4), 159.1 (s, C2), 156.8 (s, C1 of OPh), 147.9 (s, C8a), 145.4, $145.0(2 \mathrm{~s}, \mathrm{C} 1$ of $2 \mathrm{Ph}), 135.1$ (d, C7), $130.5,130.2$ ( $2 \mathrm{~d}, \mathrm{C} 3 \mathrm{of} \mathrm{OPh}), 128.4,128.3,128.1,128.0$ ( $4 \mathrm{~d}, \mathrm{C} 3$ of $2 \mathrm{Ph}), 127.2,127.1$ ( $\mathrm{d}, \mathrm{C} 5), 126.9,126.8$ (2 d, C6), 126.4, 126.3 (2 d, C8), 126.0, 125.5 (2 d, C2 of 2 $\mathrm{Ph}), 125.4,125.0(2 \mathrm{~d}, \mathrm{C} 4$ of $2 \mathrm{Ph}), 123.1,122.5(2 \mathrm{~d}, \mathrm{C} 4$ of $\mathrm{OPh}), 120.2$ (s, C4a), 116.6, 114.4 (2 d, C2 of OPh), 80.0, $79.3(2 \mathrm{~s}, \mathrm{C}-\mathrm{OH}), 75.9,74.6(2 \mathrm{~d}, \mathrm{CH}), 42.4,41.9$ $(2 \mathrm{~d}, \mathrm{CH}) 19.2,18.9\left(2 \mathrm{q}, \mathrm{CH}_{3}\right), 15.8,14.9,13.8(3 \mathrm{q}$, $\mathrm{CH}_{3}$ ).

\section{2-(2-Hydroxy-1-methyl-2-phenylethyl)-3-(2-} phenoxypropionylamino)quinazolin $4(3 H)$-one (14)

mp 90-93 ${ }^{\circ} \mathrm{C}$; four diastereoisomers (NMR).

EI-MS: $m / z(\%)=337$ (3), $216(11), 173$ (8), 130 (15), 121 (33), 94 (29), 77 (100), 39 (41).

CI-MS: $m / z(\%)=444\left(\mathrm{MH}^{+}, 10\right), 338(5), 281(41), 183$ (40), 175 (56), 122 (48), 91 (100), 74 (38).
HRMS: $m / z$ calcd for $\mathrm{C}_{26} \mathrm{H}_{26} \mathrm{~N}_{3} \mathrm{O}_{4}\left(\mathrm{MH}^{+}\right)$: 444.1923; found: 444.1926.

${ }^{1} \mathrm{H} \mathrm{NMR}\left(\mathrm{CDCl}_{3}\right): \delta=8.85,8.75,8.67,8.61$ (4 s, exch., $1 \mathrm{H}, \mathrm{NH}), 8.11-6.82(\mathrm{~m}, 14 \mathrm{H}, \mathrm{ArH}), 4.90(\mathrm{~m}, 1 \mathrm{H}, \mathrm{CH})$, $4.79,4.57(2 \mathrm{~d}, J=6 \mathrm{~Hz}, 1 \mathrm{H}, \mathrm{CH}), 4.03,3.89(2 \mathrm{br} \mathrm{s}$, exch., $1 \mathrm{H}, \mathrm{OH}$ ), 3.46, 3.15, 2.84 (3 pentet, $J=7 \mathrm{~Hz}, 1$ $\mathrm{H}, \mathrm{CH}), 1.71,1.61\left(2 \mathrm{~d}, J=7 \mathrm{~Hz}, 3 \mathrm{H}, \mathrm{CH}_{3}\right), 1.22,1.17$, $0.61\left(3 \mathrm{~d}, J=7 \mathrm{~Hz}, 3 \mathrm{H}, \mathrm{CH}_{3}\right)$.

${ }^{13} \mathrm{C} \mathrm{NMR}\left(\mathrm{CDCl}_{3}\right): \delta=172.9,172.6,172.1,171.1(4 \mathrm{~s}$, $\mathrm{C}=\mathrm{O}), 160.2,160.1,159.6(3 \mathrm{~s}, \mathrm{C} 4), 159.6,159.1(2 \mathrm{~s}$, $\mathrm{C} 2), 156.8,156.7$ (2 s, C1 of OPh), 146.3, 146.2 (2 s, C8a), 142.7, 142.3, 141.7 (3 s, C1 of Ph), 135.1, 135.0, 134.8 (3 d, C7), 128.6, 128.4 ( $2 \mathrm{~d}, \mathrm{C} 3 \mathrm{of} \mathrm{OPh}), 128.2$, 128.1 ( $2 \mathrm{~d}, \mathrm{C} 3 \mathrm{of} \mathrm{Ph}), 127.6,127.2$ ( $2 \mathrm{~d}, \mathrm{C} 5), 127.2$, 127.1 ( $2 \mathrm{~d}, \mathrm{C} 6), 127.0,126.8$ ( $2 \mathrm{~d}, \mathrm{C} 2$ of $\mathrm{Ph}), 126.2$, 125.8 (2 d, C8), 122.7, 122.5, 122.4, 122.3 (4 d, C4 of $\mathrm{Ph}), 120.9,120.7$ (2 s, C4a), 116.3 (d, C4 of OPh), 114.9, 114.6 ( $2 \mathrm{~d}, \mathrm{C} 2$ of $\mathrm{OPh}), 78.5,77.8,77.2(3 \mathrm{~d}, \mathrm{CH}), 76.1$, 75.2, 74.6, $74.3(4 \mathrm{~d}, \mathrm{CH}), 43.5,42.8,42.6,42.0(4 \mathrm{~d}$, $\mathrm{CH}) 19.0,18.9,18.4\left(3 \mathrm{q}, \mathrm{CH}_{3}\right), 16.9,16.5,16.3(3 \mathrm{q}$, $\left.\mathrm{CH}_{3}\right)$.

\section{2-(2-Hydroxy-1-methyl-2-phenylpropyl)-3-(2-} phenoxypropionylamino)quinazolin-4(3H)-one (15)

mp $148-150{ }^{\circ} \mathrm{C}$; four diastereoisomers ( ${ }^{1} \mathrm{H}$ NMR).

EI-MS: $m / z(\%)=337$ (6), $216(37), 173$ (20), 130 (15), 121 (38), 105 (53), 94 (29), 77 (100).

CI-MS: $m / z(\%)=458\left(\mathrm{MH}^{+}, 88\right), 338$ (25), 295 (100).

HRMS: $m / z$ calcd for $\mathrm{C}_{27} \mathrm{H}_{28} \mathrm{~N}_{3} \mathrm{O}_{4}\left(\mathrm{MH}^{+}\right)$: 458.2080; found: 458.2073 .

${ }^{1} \mathrm{H}$ NMR $\left(\mathrm{CDCl}_{3}\right): \delta=9.16,9.11,8.92,8.88(4 \mathrm{~s}$, exch., $1 \mathrm{H}, \mathrm{NH}), 8.23-6.90$ (m, $14 \mathrm{H}, \mathrm{ArH}), 6.51,6.15$ (2 br s, exch., $1 \mathrm{H}, \mathrm{OH}), 5.06,4.96(2 \mathrm{q}, J=7 \mathrm{~Hz}, 1 \mathrm{H}, \mathrm{CH})$, $3.50,3.30,3.20,3.00(4 \mathrm{q}, J=7 \mathrm{~Hz}, 1 \mathrm{H}, \mathrm{CH}), 1.91$, $1.89,1.78\left(3 \mathrm{~d}, J=7 \mathrm{~Hz}, 3 \mathrm{H}, \mathrm{CH}_{3}\right) 1.64,1.48,1.21(3 \mathrm{~s}$, $\left.3 \mathrm{H}, \mathrm{CH}_{3}\right), 1.11,1.07,1.02,0.60(4 \mathrm{~d}, J=7 \mathrm{~Hz}, 3 \mathrm{H}$, $\left.\mathrm{CH}_{3}\right)$.

${ }^{13} \mathrm{C}$ NMR $\left(\mathrm{CDCl}_{3}\right): \delta=172.3,172.2,171.9(3 \mathrm{~s}, \mathrm{C}=\mathrm{O})$, $162.1,161.8(2 \mathrm{~s}, \mathrm{C} 4), 159.5,159.4(2 \mathrm{~s}, \mathrm{C} 2), 157.0$, 156.7, 156.6 ( $3 \mathrm{~s}, \mathrm{C} 1$ of $\mathrm{OPh}), 146.3,146.1$ (2 s, C8a), 145.5, 145.4, 145.3 (3 s, C1 of Ph), 135.3 (d, C7), 130.2, 130.1 ( $2 \mathrm{~d}, \mathrm{C} 3$ of OPh), 129.9, 128.2, 128.1 (3 d, C3 of $\mathrm{Ph}), 127.3,127.2,127.1$ (3 d, C2 of Ph), 127.0, 126.8 (2 d, C5), 126.5, 126.4 (2 d, C6), 124.9, 124.8, 124.7 (3 d, C8), 122.9, 122.6, 122.5 (3 d, C4 of Ph), 120.5 (s, C4a), 116.5, 116.4 ( $2 \mathrm{~d}, \mathrm{C} 4$ of $\mathrm{OPh}), 114.7,114.6(2 \mathrm{~d}, \mathrm{C} 2$ of $\mathrm{OPh}), 77.4,75.8,75.4(3 \mathrm{~d}, \mathrm{CH}), 74.9,74.6(2 \mathrm{~s}, \mathrm{C}-\mathrm{OH})$, 44.6, 44.2 (2 d, $\mathrm{CH}), 30.0,29.9$ (2 q, $\left.\mathrm{CH}_{3}\right), 20.7,19.2$, $19.1,18.9\left(4 \mathrm{q}, \mathrm{CH}_{3}\right), 15.2,14.9,14.7,14.5\left(4 \mathrm{q}, \mathrm{CH}_{3}\right)$.

2-(2,2-Diphenyl-2-hydroxy-1-methylethyl)-3-[2-(1naphthalene)propionylamino]quinazolin- $4(3 H)$-one (16) 
mp 131-133 ${ }^{\circ} \mathrm{C}$; two diastereoisomers in equal proportions ( ${ }^{1} \mathrm{H}$ NMR).

EI-MS: $m / z(\%)=372$ (38), 371 (100), 356 (15), 355 (61).

CI-MS: $m / z(\%)=554\left(\mathrm{MH}^{+}, 100\right), 372(20), 200(75)$, $183(30)$.

HRMS: $m / z$ calcd for $\mathrm{C}_{36} \mathrm{H}_{32} \mathrm{~N}_{3} \mathrm{O}_{3}\left(\mathrm{MH}^{+}\right)$: 554.2444; found: 554.2444 .

${ }^{1} \mathrm{H}$ NMR $\left(\mathrm{CDCl}_{3}\right): \delta=8.45-6.50(\mathrm{~m}, 23 \mathrm{H}, \mathrm{ArH}, \mathrm{OH}$ and $\mathrm{NH}), 4.75,4.66(2 \mathrm{q}, J=7 \mathrm{~Hz}, 1 \mathrm{H}, \mathrm{CH}), 3.78,3.66(2 \mathrm{q}$, $J=7 \mathrm{~Hz}, 1 \mathrm{H}, \mathrm{CH}), 2.03,1.77\left(2 \mathrm{~d}, J=7 \mathrm{~Hz}, 3 \mathrm{H}, \mathrm{CH}_{3}\right)$, 1.26, $1.24\left(2 \mathrm{~d}, J=7 \mathrm{~Hz}, 3 \mathrm{H}, \mathrm{CH}_{3}\right)$.

${ }^{13} \mathrm{C} \mathrm{NMR}\left(\mathrm{CDCl}_{3}\right): \delta=177.3,174.9(2 \mathrm{~s}, \mathrm{C}=\mathrm{O}), 161.5$, 161.4 (2 s, C4), 159.6, 159.1 (s, C2), 147.2, 147.1 (2 s, $\mathrm{C} 8 \mathrm{a}), 145.4,145.3$ (2 s, C1 of $2 \mathrm{Ph}), 136.5$ (s, C1'), 135.2, 135.1 (2 d, C7), 134.2, 134.03 (2 s, C4'a), 132.1, 130.9 (2 s, C8'a), 129.7, 129.5 (2 d, C5'), 129.2, 128.8 (2 d, C5), 128.1, 127.8 (2 d, C3 of 2 Ph), 127.7, 127.2 (2 d, C2 of Ph), 127.1, 126.9 (2 d, C6), 126.9, 126.8 (2 d, C8), 126.5, 126.4 (2 d, C3'), 126.2, 126.0 (2 d, C2'), 126.0, 125.7 ( $2 \mathrm{~d}, \mathrm{C}^{\prime}$ ) , 125.4, 125.3 (2 d, C7'), 124.4, 124.2 (2 d, $\left.\mathrm{C6}^{\prime}\right), 124.3$ (d, C4 of $\left.2 \mathrm{Ph}\right), 123.1,122.6$ (2 d, C8'), 120.2 (s, C4a), 79.0, 78.9 (2 s, C-OH), 43.0, 42.1 (2 d, $\mathrm{CH}), 41.6,41.6(2 \mathrm{~d}, \mathrm{CH}), 17.2,17.1\left(2 \mathrm{q}, \mathrm{CH}_{3}\right), 15.0$ (q, $\mathrm{CH}_{3}$ ).

\section{2-(2,2-Diphenyl-2-hydroxy-1-methylethyl)-3-(2-} phenoxybutyrylamino)quinazolin-4(3H)-one (17)

mp 183-184 ${ }^{\circ} \mathrm{C} ; \mathbf{1 7 a} / \mathbf{1 7 b}=1: 4\left({ }^{1} \mathrm{H}\right.$ NMR).

Anal. Calcd for $\mathrm{C}_{33} \mathrm{H}_{31} \mathrm{~N}_{3} \mathrm{O}_{4}$ : C, 74.28; H, 5.86; N, 7.87. Found: C, 74.2; H, 5.7; N, 8.1.

EI-MS: $m / z(\%)=351$ (4), 216 (12), 182 (35), 135 (22), 105 (100), 77 (85), 51 (40).

CI-MS: $m / z(\%)=534\left(\mathrm{MH}^{+}, 19\right), 357(100)$.

HRMS: $m / z$ calcd for $\mathrm{C}_{33} \mathrm{H}_{32} \mathrm{~N}_{3} \mathrm{O}_{4}\left(\mathrm{MH}^{+}\right)$: 534.2393; found: 534.2393 .

\section{Compound 17a}

${ }^{1} \mathrm{H}$ NMR (DMSO- $d_{6}$ ): $\delta=11.45$ (s, exch., $1 \mathrm{H}, \mathrm{NH}$ ), $8.07(\mathrm{dd}, J=8,1 \mathrm{~Hz}, 1 \mathrm{H}, \mathrm{H} 5), 7.77-6.86$ (m, $19 \mathrm{H}, \mathrm{ArH}$ and $\mathrm{OH}), 4.94(\mathrm{t}, J=7 \mathrm{~Hz}, 1 \mathrm{H}, \mathrm{CH}), 3.99(\mathrm{q}, J=7 \mathrm{~Hz}$, $1 \mathrm{H}, \mathrm{CH}), 2.20\left(\mathrm{~m}, 2 \mathrm{H}, \mathrm{CH}_{2}\right), 1.16(\mathrm{t}, J=7 \mathrm{~Hz}, 3 \mathrm{H}$, $\left.\mathrm{CH}_{3}\right), 0.90$ (d, $\left.J=7 \mathrm{~Hz}, 3 \mathrm{H}, \mathrm{CH}_{3}\right)$.

${ }^{13} \mathrm{C}$ NMR (DMSO- $\left.d_{6}\right): \delta=171.6(\mathrm{~s}, \mathrm{C}=\mathrm{O}), 161.8(\mathrm{~s}, \mathrm{C} 4)$, 159.0 (s, C2), 157.5 (s, C1 of OPh), 148.1 (s, C8a), 145.7, 144.6 (2 s, C1 of $2 \mathrm{Ph}), 135.0$ (d, C7), 129.1 (d, C3 of OPh), 127.9, 127.7 (2 d, C3 of $2 \mathrm{Ph}), 127.0$ (d, C5), 126.5 (d, C6), 126.4 (d, C8), 125.5 (d, C4 of 2 Ph), 125.0, 124.8 (2 d, C2 of $2 \mathrm{Ph}), 121.6(\mathrm{~d}, \mathrm{C} 4$ of $\mathrm{OPh})$, 120.2 (s, C4a), 115.5 (d, C2 of OPh), 78.7 (s, C-OH), 77.7 (d, CH), 41.8 (d, CH), $26.1\left(\mathrm{t}, \mathrm{CH}_{2}\right), 14.0$ (q, $\left.\mathrm{CH}_{3}\right)$, $9.5\left(\mathrm{q}, \mathrm{CH}_{3}\right)$.

\section{Compound 17b}

${ }^{1} \mathrm{H}$ NMR (DMSO- $d_{6}$ ): $\delta=11.74$ (s, exch., $1 \mathrm{H}, \mathrm{NH}$ ), 8.07 (dd, $J=8,1 \mathrm{~Hz}, 1 \mathrm{H}, \mathrm{H} 5), 7.77-6.86$ (m, 19 H, ArH and $\mathrm{OH}), 4.82(\mathrm{t}, J=7 \mathrm{~Hz}, 1 \mathrm{H}, \mathrm{CH}), 3.95(\mathrm{q}, J=7 \mathrm{~Hz}, 1$ $\mathrm{H}, \mathrm{CH}), 2.50\left(\mathrm{~m}, 2 \mathrm{H}, \mathrm{CH}_{2}\right), 1.22\left(\mathrm{t}, J=7 \mathrm{~Hz}, 3 \mathrm{H}, \mathrm{CH}_{3}\right)$, $0.95\left(\mathrm{~d}, J=7 \mathrm{~Hz}, 3 \mathrm{H}, \mathrm{CH}_{3}\right)$.

${ }^{13} \mathrm{C}$ NMR (DMSO- $\left.d_{6}\right): \delta=172.6(\mathrm{~s}, \mathrm{C}=\mathrm{O}), 162.3$ (s, C4), 158.2 (s, C2), 157.5 (s, C1 of OPh), 148.2 (s, C8a), 145.1, 144.7 (2 s, C1 of $2 \mathrm{Ph}), 135.0$ (d, C7), 129.5 (d, C3 of OPh), 128.0, 127.8 (2 d, C3 of $2 \mathrm{Ph}), 127.0$ (d, C5), 126.5 (d, C6), 126.4 (d, C8), 125.9 (d, C4 of 2 Ph), 125.1, 124.8 (2 d, C2 of $2 \mathrm{Ph}), 121.7$ (d, C4 of OPh), 120.1 (s, C4a), 114.9 (d, C2 of OPh), 78.9 (s, C-OH), 78.8 (d, CH), 41.1 (d, CH), 26.5 (t, $\left.\mathrm{CH}_{2}\right), 14.1$ (q, $\mathrm{CH}_{3}$ ), $9.6\left(\mathrm{q}, \mathrm{CH}_{3}\right)$.

\section{2-(2-Hydroxy-1-methyl-2-phenylethyl)-3-(2- phenoxybutyrylamino)quinazolin-4(3H)-one (18)}

mp 100-102 ${ }^{\circ} \mathrm{C}$; four diastereoisomers ( ${ }^{1} \mathrm{H}$ NMR).

EI-MS: $m / z(\%)=230(6), 216$ (28), 173 (32), 135 (44), 105 (49), 94 (33), 77 (100).

CI-MS: $m / z(\%)=458\left(\mathrm{MH}^{+}, 33 \%\right), 281(70), 197(40)$, 175 (53), 136 (46), 114 (41), 105 (100), 94 (56).

HRMS: $m / z$ calcd for $\mathrm{C}_{27} \mathrm{H}_{28} \mathrm{~N}_{3} \mathrm{O}_{4}\left(\mathrm{MH}^{+}\right)$: 458.2080; found: 458.2074 .

${ }^{1} \mathrm{H}$ NMR $\left(\mathrm{CDCl}_{3}\right): \delta=9.19,9.08,8.98,8.72$ (4 s, exch., $1 \mathrm{H}, \mathrm{NH}), 8.04-6.51$ (m, $14 \mathrm{H}, \mathrm{ArH}), 5.65,5.52,5.30$, 5.20 (4 s, exch., $1 \mathrm{H}, \mathrm{OH}), 4.79-4.19$ (m, $2 \mathrm{H}, 2 \mathrm{CH})$, $3.37,3.02,2.71,2.51$ (4 pentet, $J=7 \mathrm{~Hz}, 1 \mathrm{H}, \mathrm{CH}$ ), 2.24-1.98 (m, $\left.2 \mathrm{H}, \mathrm{CH}_{2}\right), 1.32-0.96\left(\mathrm{~m}, 3 \mathrm{H}, \mathrm{CH}_{3}\right), 0.91$, $0.87,0.46,0.35$ (4 d, $\left.J=7 \mathrm{~Hz}, 3 \mathrm{H}, \mathrm{CH}_{3}\right)$.

${ }^{13} \mathrm{C} \mathrm{NMR}\left(\mathrm{CDCl}_{3}\right): \delta=171.2,171.0(2 \mathrm{~s}, \mathrm{C}=\mathrm{O}), 161.0$, 160.1 (2 s, C4), 159.0, 158.7 (2 s, C2), 157.5, 157.4 (2 s, $\mathrm{C} 1$ of $\mathrm{OPh}$ ), 145.2, 145.0 (2 s, C8a), 143.9, 143.6, 143.6 (3 s, C1 of Ph), 135.1 (d, C7), 129.6 (d, C3 of OPh), 127.9, 127.8 (2 d, C3 of Ph), 127.7 (d, C5), 127.2 (d, C6), 127.0, 126.7 (2 d, C2 of Ph), 126.6, 125.4 (2 d, C4 of $\mathrm{Ph}), 126.3$ (d, C8), 121.6, 121.5 (2 d, C4 of OPh), 121.4 (s, C4a), 115.6, 115.4, 115.1 (3 d, C2 of OPh), 78.2, 78.0, 77.4 (3 d, CH), 73.7, 72.9 (2 d, CH), 42.9, $42.6(2 \mathrm{~d}, \mathrm{CH}) 26.1,26.0,25.8\left(3 \mathrm{t}, \mathrm{CH}_{2}\right), 13.5,13.2$, $13.1\left(3 \mathrm{q}, \mathrm{CH}_{3}\right), 9.4,9.3\left(2 \mathrm{q}, \mathrm{CH}_{3}\right)$.

\section{2-(2-Hydroxy-1-methyl-2-phenylpropyl)-3-(2-} phenoxybutyrylamino)quinazolin-4(3H)-one (19)

mp 157-158 ${ }^{\circ} \mathrm{C}$; four diastereoisomers (NMR).

EI-MS: $m / z(\%)=351$ (54), 258 (29), 220 (52), 216 (100), 202 (98), 189 (64).

EI-MS: $m / z(\%)=472\left(\mathrm{MH}^{+}, 6\right), 295(12), 175$ (23), 138 (100), 105 (32).

HRMS: $m / z$ calcd for $\mathrm{C}_{28} \mathrm{H}_{30} \mathrm{~N}_{3} \mathrm{O}_{4}\left(\mathrm{MH}^{+}\right)$: 472.2236 ; found: 472.2239 . 
${ }^{1} \mathrm{H} \mathrm{NMR}\left(\mathrm{CDCl}_{3}\right): \delta=8.91,8.89,8.76,8.71(4 \mathrm{~s}, \mathrm{exch}$. $1 \mathrm{H}, \mathrm{NH}), 8.15-6.79(\mathrm{~m}, 14 \mathrm{H}, \mathrm{ArH}), 5.60,6.04(2 \mathrm{br} \mathrm{s}$, exch., $1 \mathrm{H}, \mathrm{OH}), 4.79-4.63(\mathrm{~m}, 1 \mathrm{H}, \mathrm{CH}), 3.48,3.21$, 3.11, $2.90(4 \mathrm{q}, J=7 \mathrm{~Hz}, 1 \mathrm{H}, \mathrm{CH}), 2.14-1.98(\mathrm{~m}, 2 \mathrm{H}$, $\left.\mathrm{CH}_{2}\right), 1.56,1.54,1.37,1.35\left(4 \mathrm{~s}, 3 \mathrm{H}, \mathrm{CH}_{3}\right), 1.15,1.13(2$ $\left.\mathrm{t}, J=7 \mathrm{~Hz}, 3 \mathrm{H}, \mathrm{CH}_{3}\right), 0.92,0.51(2 \mathrm{~d}, J=7 \mathrm{~Hz}, 3 \mathrm{H}$, $\left.\mathrm{CH}_{3}\right)$.

${ }^{13} \mathrm{C}$ NMR $\left(\mathrm{CDCl}_{3}\right): \delta=172.9,172.8,172.6(3 \mathrm{~s}, \mathrm{C}=\mathrm{O})$, $162.1,161.9,161.8$ (3 s, C4), 159.5, $159.4(2 \mathrm{~s}, \mathrm{C} 2)$, 157.4, 157.1 ( $2 \mathrm{~s}, \mathrm{Cl}$ of $\mathrm{OPh}), 146.2,146.1$ (2 s, C8a), 145.5, 145.3 (2 s, C1 of Ph), 135.3 (d, C7), 130.2, 130.1, 130.0 (3 d, C3 of OPh), 128.2, 128.1 ( $2 \mathrm{~d}, \mathrm{C} 3 \mathrm{of} \mathrm{Ph})$, 127.3, 127.2 ( 2 d, C2 of Ph), 127.0 (d, C5), 126.5, 126.4 (2 d, C6), 124.9, 124.8 (2 d, C8), 123.1, 122.6, 122.4 (3 d, C4 of Ph), 120.6 (s, C4a), 116.8, 116.7 (2 d, C4 of $\mathrm{OPh}), 114.7,114.6$ (2 d, C2 of OPh), 81.0, 80.8, 79.9, 79.6 (4 d, CH), 75.5, 75.4 ( $2 \mathrm{~s}, \mathrm{C}-\mathrm{OH}), 44.7,44.6,44.2$ $(3 \mathrm{~d}, \mathrm{CH}), 30.0,29.8\left(2 \mathrm{q}, \mathrm{CH}_{3}\right), 26.9,26.8,26.2$ (3 t, $\left.\mathrm{CH}_{2}\right), 15.0,14.9,14.4\left(3 \mathrm{q}, \mathrm{CH}_{3}\right), 9.8,9.7,9.0(3 \mathrm{q}$, $\mathrm{CH}_{3}$ ).

\section{2-(2,2-Diphenyl-2-hydroxy-1-methylethyl)-3-(2-} phenylbutyrylamino)quinazolin-4(3H)-one (20)

mp $182-183{ }^{\circ} \mathrm{C} ; \mathbf{2 0 a} / \mathbf{2 0 b}=1: 4\left({ }^{1} \mathrm{H}\right.$ NMR).

Anal. Calcd for $\mathrm{C}_{33} \mathrm{H}_{31} \mathrm{~N}_{3} \mathrm{O}_{3}$ : C, 76.59; $\mathrm{H}, 6.00 ; \mathrm{N}, 8.12$. Found: C, 76.6; H, 6.0; N, 8.1.

CI-MS: $m / z(\%)=518\left(\mathrm{MH}^{+}, 90\right), 357(12), 336(100)$, 200 (22), 183 (50), 175 (33), 105 (21).

HRMS: $m / z$ calcd for $\mathrm{C}_{33} \mathrm{H}_{32} \mathrm{~N}_{3} \mathrm{O}_{3}\left(\mathrm{MH}^{+}\right)$: 518.2444; found: 518.2444 .

\section{Compound 20a}

${ }^{1} \mathrm{H}$ NMR (DMSO- $d_{6}$ ): $\delta=11.71$ (br s, exch., $1 \mathrm{H}, \mathrm{NH}$ ), 8.14 (dd, $J=8,1 \mathrm{~Hz}, 1 \mathrm{H}, \mathrm{H} 5), 7.77-6.86(\mathrm{~m}, 18 \mathrm{H}$, ArH), 6.73 (s, exch., $1 \mathrm{H}, \mathrm{OH}), 4.12(\mathrm{q}, J=7 \mathrm{~Hz}, 1 \mathrm{H}$, $\mathrm{CH}), 3.66(\mathrm{t}, J=7 \mathrm{~Hz}, 1 \mathrm{H}, \mathrm{CH}), 2.34-2.07(\mathrm{~m}, 2 \mathrm{H}$, $\left.\mathrm{CH}_{2}\right), 1.33\left(\mathrm{~d}, J=7 \mathrm{~Hz}, 3 \mathrm{H}, \mathrm{CH}_{3}\right), 1.07(\mathrm{t}, J=7 \mathrm{~Hz}, 3$ $\mathrm{H}, \mathrm{CH}_{3}$ ).

${ }^{13} \mathrm{C}$ NMR (DMSO- $\left.d_{6}\right): \delta=172.8(\mathrm{~s}, \mathrm{C}=\mathrm{O}), 162.4(\mathrm{~s}, \mathrm{C} 4)$, 158.6 (s, C2), 148.5 (s, C8a), 146.2, $145.1(2 \mathrm{~s}, \mathrm{C} 1$ of Ph's), 135.2 (d, C7), 129.5, 128.3, 128.1 (3 d, C3 of Ph's), 128.0 (d, C5), 127.4 (d, C6), 127.3 (d, C8), 126.6, 126.0 ( $2 \mathrm{~d}, \mathrm{C} 4$ of Ph's), 125.8, 125.7, 125.4 (3 d, C2 of Ph's), 120.3 (s, C4a), 79.1 (s, C-OH), 52.3 (d, CH), 41.6 (d, $\mathrm{CH}), 25.8\left(\mathrm{t}, \mathrm{CH}_{2}\right), 14.3\left(\mathrm{q}, \mathrm{CH}_{3}\right), 12.7\left(\mathrm{q}, \mathrm{CH}_{3}\right)$.

\section{Compound 20b}

${ }^{1} \mathrm{H}$ NMR (DMSO- $d_{6}$ ): $\delta=11.71$ (br s, exch., $1 \mathrm{H}, \mathrm{NH}$ ), $8.12(\mathrm{dd}, J=8,1 \mathrm{~Hz}, 1 \mathrm{H}, \mathrm{H} 5), 7.77-6.86(\mathrm{~m}, 18 \mathrm{H}$, ArH), 6.73 (s, exch., $1 \mathrm{H}, \mathrm{OH}), 3.86(\mathrm{q}, J=7 \mathrm{~Hz}, 1 \mathrm{H}$, $\mathrm{CH}), 3.63(\mathrm{t}, J=7 \mathrm{~Hz}, 1 \mathrm{H}, \mathrm{CH}), 2.28-1.87(\mathrm{~m}, 2 \mathrm{H}$, $\left.\mathrm{CH}_{2}\right), 1.21\left(\mathrm{~d}, J=7 \mathrm{~Hz}, 3 \mathrm{H}, \mathrm{CH}_{3}\right), 0.90(\mathrm{t}, J=7 \mathrm{~Hz}, 3$ $\mathrm{H}, \mathrm{CH}_{3}$ ).

${ }^{13} \mathrm{C}$ NMR (DMSO- $\left.d_{6}\right): \delta=173.3(\mathrm{~s}, \mathrm{C}=\mathrm{O}), 162.7(\mathrm{~s}, \mathrm{C} 4)$, 158.5 (s, C2), 148.3 (s, C8a), 145.9, $145.0(\mathrm{~s}, \mathrm{C} 1$ of Ph's), 135.4 (d, C7), 129.2, 128.8, 128.5 (3 d, C3 of
Ph's), 127.8 (d, C5), 127.6 (d, C6), 126.8 (d, C8), 126.3, 126.1 ( $2 \mathrm{~d}, \mathrm{C} 4$ of Ph's), 125.0, 124.8, 124.7 (3 d, C2 of Ph's), 120.1 (s, C4a), 79.0 (s, C-OH), 51.6 (d, CH), 41.6 $(\mathrm{d}, \mathrm{CH}), 24.7\left(\mathrm{t}, \mathrm{CH}_{2}\right), 14.0\left(\mathrm{q}, \mathrm{CH}_{3}\right), 12.4\left(\mathrm{q}, \mathrm{CH}_{3}\right)$.

\section{2-(1,2-Dimethyl-2-hydroxybutyl)-3-(2- phenylbutyrylamino)quinazolin-4(3H)-one (21)}

mp 90-93 ${ }^{\circ} \mathrm{C}$; at least seven diastereoisomers ( ${ }^{1} \mathrm{H}$ NMR). CI-MS: $m / z(\%)=408\left(\mathrm{MH}^{+}, 93\right), 336$ (58), 273 (12), 247 (84), 175 (100).

HRMS: $m / z$ calcd for $\mathrm{C}_{24} \mathrm{H}_{30} \mathrm{~N}_{3} \mathrm{O}_{3}\left(\mathrm{MH}^{+}\right)$: 408.2287; found: 408.2287 .

${ }^{1} \mathrm{H}$ NMR $\left(\mathrm{CDCl}_{3}\right): \delta=8.94,8.84,8.83,8.76,8.64,8.46$, 8.39 ( 7 s, exch., $1 \mathrm{H}, \mathrm{NH}), 8.19$ (m, $1 \mathrm{H}, \mathrm{H} 5), 7.77-6.90$ $(\mathrm{m}, 8 \mathrm{H}, \mathrm{ArH}), 6.13,6.06,5.81,5.67,5.23,5.10,4.56$ (7 s, exch., $1 \mathrm{H}, \mathrm{OH}), 3.65-3.58(\mathrm{~m}, 1 \mathrm{H}, \mathrm{CH}), 3.11,2.88$, 2.74, 2.70, 2.59, 2.46, $2.37(7 \mathrm{q}, J=7 \mathrm{~Hz}, 1 \mathrm{H}, \mathrm{CH})$, 2.30-2.18 (m, $\left.2 \mathrm{H}, \mathrm{CH}_{2}\right), 1.92,1.88,1.85,1.80(4 \mathrm{~s}, 3 \mathrm{H}$, $\left.\mathrm{CH}_{3}\right), 1.69-0.52\left(\mathrm{~m}, 11 \mathrm{H}, \mathrm{CH}_{2}\right.$ and $\left.3 \mathrm{CH}_{3}\right)$.

${ }^{13} \mathrm{C}$ NMR $\left(\mathrm{CDCl}_{3}\right): \delta=174.3,174.1,173.2(3 \mathrm{~s}, \mathrm{C}=\mathrm{O})$, $162.7,162.5,161.9,161.7$ (4 s, C4), 160.3, 160.1, 160.0 (3 s, C2), 146.5, 146.3 (2 s, C8a), 138.9, 138.8, 138.7 (3 s, C1 of Ph), 135.1, 135.0 (2 d, C7), 129.0, 128.9 (2 d, $\mathrm{C} 3 \mathrm{of} \mathrm{Ph}), 128.1,128.0(2 \mathrm{~d}, \mathrm{C} 2 \mathrm{of} \mathrm{Ph}), 127.9,127.8$ (2 d, C5), 127.7, 127.6 (2 d, C6), 127.3, 127.2 (2 d, C8), $127.1,127.0,126.9$ ( $3 \mathrm{~d}, \mathrm{C} 4 \mathrm{of} \mathrm{Ph}), 120.5,120.4(2 \mathrm{~s}$, C4a), 74.6, 73.9, 73.8, 73.6 (4 s, C-OH), 53.3, 52.9, 52.8 $(3 \mathrm{~d}, \mathrm{CH}), 41.4,41.1,40.6,40.4(4 \mathrm{~d}, \mathrm{CH}), 34.4,33.8$, $31.9,26.8,25.7,25.6\left(6 \mathrm{t}, 2 \mathrm{CH}_{2}\right), 23.0,22.6,20.0(3 \mathrm{q}$, $\left.\mathrm{CH}_{3}\right), 14.3,14.1,13.7,13.5\left(4 \mathrm{q}, \mathrm{CH}_{3}\right), 12.4,12.1,12.0$, $10.6\left(4 \mathrm{q}, \mathrm{CH}_{3}\right), 8.4,8.2,8.1,7.7\left(4 \mathrm{q}, \mathrm{CH}_{3}\right)$.

\section{2-(1-Diphenylhydroxymethylpropyl)-3-(2- phenoxypropionylamino)quinazolin- $4(3 \mathrm{H})$-one (22)}

mp $112-114{ }^{\circ} \mathrm{C}$; four diastereoisomers (NMR).

EI-MS: $m / z(\%)=369$ (12), 351 (43), 173 (18), 323 (100), 258 (44).

CI-MS: $m / z(\%)=534\left(\mathrm{MH}^{+}, 33\right), 371(19), 352(100)$, 260 (16), 217 (18).

HRMS: $m / z$ calcd for $\mathrm{C}_{33} \mathrm{H}_{32} \mathrm{~N}_{3} \mathrm{O}_{4}\left(\mathrm{MH}^{+}\right)$: 534.2393; found: 534.2397 .

${ }^{1} \mathrm{H}$ NMR (DMSO- $d_{6}$ ): $\delta=11.47,11.24$ ( 2 br s, exch., 1 $\mathrm{H}, \mathrm{NH}), 8.07$ (m, $1 \mathrm{H}, \mathrm{H} 5), 7.79-6.83(\mathrm{~m}, 19 \mathrm{H}, \mathrm{ArH}$ and $\mathrm{OH}), 5.35,5.24(2 \mathrm{q}, J=7 \mathrm{~Hz}, 1 \mathrm{H}, \mathrm{CH}), 4.28,4.06$, 3.96, $3.84(4 \mathrm{t}, J=7.5 \mathrm{~Hz}, 1 \mathrm{H}, \mathrm{CH}), 1.98-1.44(\mathrm{~m}, 2 \mathrm{H}$, $\left.\mathrm{CH}_{2}\right), 1.82,1.78,1.75,1.68\left(4 \mathrm{~d}, J=7 \mathrm{~Hz}, 3 \mathrm{H}, \mathrm{CH}_{3}\right)$, $0.71,0.41,0.35,0.30\left(4 \mathrm{t}, J=7.5 \mathrm{~Hz}, 3 \mathrm{H}, \mathrm{CH}_{3}\right)$.

${ }^{13} \mathrm{C}$ NMR (DMSO- $\left.d_{6}\right): \delta=173.5,173.3,172.6,172.4(4$ $\mathrm{s}, \mathrm{C}=\mathrm{O}), 161.8,161.7,161.4(3 \mathrm{~s}, \mathrm{C} 4), 158.3,158.2$, $158.1,158.0(4 \mathrm{~s}, \mathrm{C} 2), 157.3,157.2,157.1(3 \mathrm{~s}, \mathrm{C} 1$ of $\mathrm{OPh}), 148.6,148.5,148.4,148.3$ (4 s, C8a) 145.7 , 145.4, 144.7, 144. 5 (4 s, C1 of $2 \mathrm{Ph}), 135.5,135.4(2 \mathrm{~d}$, C7), 129.9, 129.8, 129.7 (3 d, C3 of OPh), 128.3, 128.0 
( $2 \mathrm{~d}, \mathrm{C} 3$ of $2 \mathrm{Ph}), 127.9,127.8,127.5$ (3 d, C2 of $2 \mathrm{Ph})$, 126.8, 126.5 (2 d, C5), 126.2, 126.1 (2 d, C6), 125.5, 125.3 (2 d, C8), 125.1, 125.0 (2 d, C4 of $2 \mathrm{Ph}), 121.9$, 121.6 ( $2 \mathrm{~d}, \mathrm{C} 4$ of $\mathrm{OPh}), 119.9,119.8$ (2 s, C4a), 115.4, $115.3,114.6$ ( $3 \mathrm{~d}, \mathrm{C} 2$ of $\mathrm{OPh}), 79.7,79.5,79.2$ (3 s, C$\mathrm{OH}), 73.6,73.4,71.8(3 \mathrm{~d}, \mathrm{CH}), 47.9,74.5,47.3,47.1$ (4 d, CH), 22.9, 22.7 (2 t, $\left.\mathrm{CH}_{2}\right), 19.4,19.1,18.8,18.5$ (4 q, $\left.\mathrm{CH}_{3}\right), 12.6,12.3,12.1,12.0\left(4 \mathrm{q}, \mathrm{CH}_{3}\right)$.

\section{2-(1-Phenylhydroxymethylpropyl)-3-(2-} phenoxypropionylamino)quinazolin- $4(3 H)$-one (23) mp 158-159 ${ }^{\circ} \mathrm{C} ; \mathbf{2 3 a} / \mathbf{2 3 b}=3: 5\left({ }^{1} \mathrm{H}\right.$ NMR).

EI-MS: $m / z(\%)=323(10), 230(24), 187$ (12), 160 (23), 121 (44), 77 (100).

CI-MS: $m / z(\%)=458\left(\mathrm{MH}^{+}, 9\right), 295(48), 189(53), 183$ (46), 166 (17), 122 (52), 106 (41), 91 (100).

HRMS: $m / z$ calcd for $\mathrm{C}_{27} \mathrm{H}_{28} \mathrm{~N}_{3} \mathrm{O}_{4}\left(\mathrm{MH}^{+}\right)$: 458.2080 ; found: 458.2075 .

\section{Compound 23a}

${ }^{1} \mathrm{H}$ NMR (DMSO- $d_{6}$ ): $\delta=11.15$ (br s, exch., $1 \mathrm{H}, \mathrm{NH}$ ), 7.94-6.75 (m, $14 \mathrm{H}, \mathrm{ArH}), 4.91$ (q, $J=7 \mathrm{~Hz}, 1 \mathrm{H}, \mathrm{CH}$ ), $4.79(\mathrm{~d}, J=3.5 \mathrm{~Hz}$, exch., $1 \mathrm{H}, \mathrm{OH}), 4.74(\mathrm{~d}, J=5 \mathrm{~Hz}, 1$ $\mathrm{H}, \mathrm{CHOH}), 2.87(\mathrm{~m}, 1 \mathrm{H}, \mathrm{CH}), 1.63(\mathrm{~m}, 1 \mathrm{H}, 1 \mathrm{H}$ of $\left.\mathrm{CH}_{2}\right), 1.48$ (d, $\left.J=7 \mathrm{~Hz}, 3 \mathrm{H}, \mathrm{CH}_{3}\right), 1.32(\mathrm{~m}, 1 \mathrm{H}, 1 \mathrm{H}$ of $\left.\mathrm{CH}_{2}\right), 0.40\left(\mathrm{t}, J=7.5 \mathrm{~Hz}, 3 \mathrm{H}, \mathrm{CH}_{3}\right)$.

${ }^{13} \mathrm{C}$ NMR (DMSO- $\left.d_{6}\right): \delta=172.0(\mathrm{~s}, \mathrm{C}=\mathrm{O}), 159.5$ (s, C4), 158.8 (s, C2), 157.1 (s, C1 of $\mathrm{OPh}), 145.9$ (s, C8a), 143.9 (s, C1 of Ph), 135.1 (d, C7), 129.6 (d, C3 of OPh), 127.7 (d, C3 of Ph), 127.3 (d, C5), 127.0 (d, C2 of Ph), 126.7 (d, C6), 126.4 (d, C8), 126.1 (d, C4 of Ph), 121.5 (d, C4 of OPh), 120.4 (s, C4a), 115.1 (d, C2 of OPh), 73.2 (d, CH), 72.1 (d, CH), 50.1 (d, CH), 20.5 (t, $\mathrm{CH}_{2}$ ), 19.1 (q, $\left.\mathrm{CH}_{3}\right), 10.6$ (q, $\left.\mathrm{CH}_{3}\right)$.

\section{Compound 23b}

${ }^{1} \mathrm{H}$ NMR (DMSO- $d_{6}$ ): $\delta=11.15$ (br s, exch., $1 \mathrm{H}, \mathrm{NH}$ ), 7.94-6.75 (m, $14 \mathrm{H}, \mathrm{ArH}), 5.15$ (d, $J=3.5 \mathrm{~Hz}$, exch., 1 $\mathrm{H}, \mathrm{OH}), 5.00$ (q, $J=7 \mathrm{~Hz}, 1 \mathrm{H}, \mathrm{CH}), 4.88(\mathrm{~d}, J=4.5 \mathrm{~Hz}$, $1 \mathrm{H}, \mathrm{CHOH}), 2.98(\mathrm{~m}, 1 \mathrm{H}, \mathrm{CH}), 1.86(\mathrm{~m}, 1 \mathrm{H}, 1 \mathrm{H}$ of $\left.\mathrm{CH}_{2}\right), 1.43\left(\mathrm{~d}, J=7 \mathrm{~Hz}, 3 \mathrm{H}, \mathrm{CH}_{3}\right), 1.23(\mathrm{~m}, 1 \mathrm{H}, 1 \mathrm{H}$ of $\left.\mathrm{CH}_{2}\right), 0.34\left(\mathrm{t}, J=7.5 \mathrm{~Hz}, 3 \mathrm{H}, \mathrm{CH}_{3}\right)$.

${ }^{13} \mathrm{C}$ NMR (DMSO- $\left.d_{6}\right): \delta=171.4(\mathrm{~s}, \mathrm{C}=\mathrm{O}), 159.2(\mathrm{~s}, \mathrm{C} 4)$, 158.4 (s, C2), 157.0 (s, C1 of OPh), 146.3 (s, C8a), 144.1 (s, C1 of Ph), 135.0 (d, C7), 129.5 (d, C3 of OPh), 127.9 (d, C3 of Ph), 127.5 (d, C5), 126.9 (d, C2 of Ph), 126.6 (d, C6), 126.3 (d, C8), 126.2 (d, C4 of Ph), 121.6 (d, C4 of OPh), 120.6 (s, C4a), 115.5 (d, C2 of OPh), $73.0(\mathrm{~d}, \mathrm{CH}), 72.2(\mathrm{~d}, \mathrm{CH}), 50.2(\mathrm{~d}, \mathrm{CH}), 18.7\left(\mathrm{t}, \mathrm{CH}_{2}\right)$, $18.5\left(\mathrm{q}, \mathrm{CH}_{3}\right), 11.7$ (q, $\left.\mathrm{CH}_{3}\right)$.

2-(1-Ethyl-2-hydroxy-2-phenylpropyl)-3-(2phenoxypropionylamino)quinazolin-4(3H)-one (24)

$\mathrm{mp} 79-81{ }^{\circ} \mathrm{C}$; four diastereoisomers (NMR).

EI-MS: $m / z(\%)=323$ (7), 230 (22), 173 (18), 160 (40), 121 (32), 105 (49), 94 (22), 77 (100).
CI-MS: $m / z(\%)=472\left(\mathrm{MH}^{+}, 5\right), 352(17), 309(18), 189$ (76), 138 (100), 122 (22), 91 (49).

HRMS: $m / z$ calcd for $\mathrm{C}_{27} \mathrm{H}_{28} \mathrm{~N}_{3} \mathrm{O}_{4}\left(\mathrm{MH}^{+}\right)$: 472.2235; found: 472.2235 .

${ }^{1} \mathrm{H}$ NMR (DMSO- $d_{6}$ ): $\delta=11.35$ (br s, exch., $1 \mathrm{H}, \mathrm{NH}$ ), 8.23-6.78 (m, 14 H, ArH), 6.19, 6.11, 5.98 (3 s, exch., 1 $\mathrm{H}, \mathrm{OH}), 5.42-5.04(\mathrm{~m}, 1 \mathrm{H}, \mathrm{CH}), 2.67-2.33(\mathrm{~m}, 1 \mathrm{H}$, $\mathrm{CH}), 1.92-1.87\left(\mathrm{~m}, 1 \mathrm{H}, 1 \mathrm{H}\right.$ of $\left.\mathrm{CH}_{2}\right), 1.81,1.79,1.75$, $1.66\left(4 \mathrm{~d}, J=7 \mathrm{~Hz}, 3 \mathrm{H}, \mathrm{CH}_{3}\right), 1.72,1.70,1.69,1.66$ (4 $\left.\mathrm{s}, \mathrm{CH}_{3}\right), 1.17\left(\mathrm{~m}, 1 \mathrm{H}, 1 \mathrm{H}\right.$ of $\left.\mathrm{CH}_{2}\right), 0.95,0.76,0.51,0.37$ $\left(4 \mathrm{t}, J=7.5 \mathrm{~Hz}, 3 \mathrm{H}, \mathrm{CH}_{3}\right)$.

${ }^{13} \mathrm{C}$ NMR (DMSO- $\left.d_{6}\right): \delta=172.4,171.8,171.3,170.8(4$ $\mathrm{s}, \mathrm{C}=\mathrm{O}), 162.1,162.0,160.6,160.2$ (4 s, C4), 159.0, 158.9, 158.8, 158.5 (4 s, C2), 158.2, 158.1, 157.2, 157.0 (4 s, C1 of OPh), 149.3, 147.0, 146.7, 146.6 (4 s, C8a), 146.3, 145.7, 145.6, 145.1 (4 s, C1 of Ph), 135.5, 135.3, 135.0, 134.6 (4 d, C7), 129.6, 129.5, 129.3, 128.1 (4 d, $\mathrm{C} 3$ of $\mathrm{OPh}), 128.0,127.9,127.8,127.5$ (4 d, C3 of Ph), 127.4, 127.3, 127.2, 127.1 (4 d, C5), 126.9, 126.8, 126.7, 126.6 (4 d, C2 of Ph), 126.4, 126.3, 126.1, 125.9 (4 d, C6), 125.1, 124.8 (2 d, C8), 124.7, 124.6 (2 d, C4 of Ph), $121.7,121.6,121.5,121.1$ (4 d, C4 of $\mathrm{OPh}), 120.8$, 120.6, 120.4, 120.3 (4 s, C4a), 115.5, 115.2, 115.0, 114.9 (4 d, C2 of OPh), 76.3, 76.2, 75.5, 75.4 (4 s, C-OH), 73.4, 72.6, 72.0, $71.2(4 \mathrm{~d}, \mathrm{CH}), 53.7,52.1,51.4,50.3$ (4 d, $\mathrm{CH}), 31.0,30.8,30.0,29.7$ (4 q, $\left.\mathrm{CH}_{3}\right), 22.3,22.0$, 21.4, 20.9 (4 t, $\mathrm{CH}_{2}$ ), 19.3, 19.2, 19.0, 18.7 (4 q, $\mathrm{CH}_{3}$ ), $13.7,13.5,12.3,12.0\left(4 \mathrm{q}, \mathrm{CH}_{3}\right)$.

\section{2-(1-Diphenylhydroxymethylpropyl)-3-(2- phenoxybutyrylamino)quinazolin-4(3H)-one (25)}

mp $139-140{ }^{\circ} \mathrm{C}$; four diastereoisomers (NMR)

EI-MS: $m / z(\%)=365$ (51), 337 (100), 308 (20), 277 (38).

CI-MS: $m / z(\%)=548\left(\mathrm{MH}^{+}, 41\right), 371(89), 366(100)$.

HRMS: $m / z$ calcd for $\mathrm{C}_{34} \mathrm{H}_{34} \mathrm{~N}_{3} \mathrm{O}_{4}\left(\mathrm{MH}^{+}\right)$: 548.2549; found: 548.2549 .

${ }^{1} \mathrm{H}$ NMR (DMSO- $\left.d_{6}\right): \delta=11.55,11.51,11.45,11.28(4$ s, exch., $1 \mathrm{H}, \mathrm{NH}), 8.10-6.85(\mathrm{~m}, 20 \mathrm{H}, \mathrm{ArH}$ and $\mathrm{OH})$, 5.11, 5.02 (2 t, $J=7 \mathrm{~Hz}, 1 \mathrm{H}, \mathrm{CH}), 4.26,4.05,3.93,3.82$ (4 t, $J=7.5 \mathrm{~Hz}, 1 \mathrm{H}, \mathrm{CH}), 2.22-1.43\left(\mathrm{~m}, 4 \mathrm{H}, 2 \mathrm{CH}_{2}\right)$, 1.20, 1.09 ( $\left.2 \mathrm{t}, J=7 \mathrm{~Hz}, 3 \mathrm{H}, \mathrm{CH}_{3}\right), 0.68,0.34,0.30$, $0.28\left(4 \mathrm{t}, J=7.5 \mathrm{~Hz}, 3 \mathrm{H}, \mathrm{CH}_{3}\right)$.

${ }^{13} \mathrm{C}$ NMR (DMSO- $\left.d_{6}\right): \delta=172.8,171.7,171.6$ (3 s, $\mathrm{C}=\mathrm{O}), 161.8,161.3$ (2 s, C4), 158.2, 158.0 (2 s, C2), 157.7, 157.6 (2 s, C1 of OPh), 148.6, 148.5 (2 s, C8a), 145.7, 144.4 (2 s, C1 of $2 \mathrm{Ph}), 135.5,135.4$ (2 d, C7), 129.9, 129.7 (2 d, C3 of OPh), 128.3, 128.1 (2 d, C3 of 2 $\mathrm{Ph}), 127.9,127.5$ (2 d, C5), 126.8, 126.7 (2 d, C6), 126.4, 125.5 (2 d, C8), 126.1, 125.5 (2 d, C4 of $2 \mathrm{Ph})$, 125.3, 125.1 ( $2 \mathrm{~d}, \mathrm{C} 2$ of $2 \mathrm{Ph}), 121.7,121.6$ (2 d, C4 of $\mathrm{OPh}), 119.9$ (s, C4a), 115.5, 114.7 (2 d, C2 of OPh), 79.7, 79.2 (2 s, C-OH), 78.7, 76.9 (2 d, CH), 47.9, 47.8, 47.7 (3 d, CH), 26.3, $25.8\left(2 \mathrm{t}, \mathrm{CH}_{2}\right), 22.8,22.7(2 \mathrm{t}$, $\left.\mathrm{CH}_{2}\right), 12.7,12.0$ (2 q, $\left.\mathrm{CH}_{3}\right), 9.7,9.6,9.5,9.4$ (4 q, $\left.\mathrm{CH}_{3}\right)$. 
2-(1-Phenylhydroxymethylpropyl)-3-(2phenoxybutyrylamino)quinazolin-4(3H)-one (26)

mp 136-137 ${ }^{\circ} \mathrm{C} ; \mathbf{2 6 a} / \mathbf{2 6 b}=3: 5\left({ }^{1} \mathrm{H}\right.$ NMR).

EI-MS: $m / z(\%)=365$ (62), 337 (100), 308 (22), 272 (45).

CI-MS: $m / z(\%)=472\left(\mathrm{MH}^{+}, 16\right), 295$ (33), $197(70)$, 189 (100), 180 (18), 139 (18), 136 (23), 105 (82), 88 (27).

HRMS: $m / z$ calcd for $\mathrm{C}_{27} \mathrm{H}_{28} \mathrm{~N}_{3} \mathrm{O}_{4}\left(\mathrm{MH}^{+}\right)$: 472.2235; found: 472.2230 .

\section{Compound 26a}

${ }^{1} \mathrm{H}$ NMR (DMSO- $d_{6}$ ): $\delta=11.26$ (br s, exch., $1 \mathrm{H}, \mathrm{NH}$ ), 8.13-6.97 (m, $15 \mathrm{H}, \mathrm{ArH}$ and $\mathrm{OH}), 5.11(\mathrm{~d}, J=4 \mathrm{~Hz}, 1$ $\mathrm{H}, \mathrm{CHOH}), 4.94(\mathrm{t}, J=7 \mathrm{~Hz}, 1 \mathrm{H}, \mathrm{CH}), 3.08(\mathrm{~m}, 1 \mathrm{H}$, $\mathrm{CH}), 2.14-1.38\left(\mathrm{~m}, 4 \mathrm{H}, 2 \mathrm{CH}_{2}\right), 1.17(\mathrm{t}, J=7 \mathrm{~Hz}, 3 \mathrm{H}$, $\left.\mathrm{CH}_{3}\right), 0.58\left(\mathrm{t}, J=7.5 \mathrm{~Hz}, 3 \mathrm{H}, \mathrm{CH}_{3}\right)$.

${ }^{13} \mathrm{C}$ NMR (DMSO- $\left.d_{6}\right): \delta=171.3(\mathrm{~s}, \mathrm{C}=\mathrm{O}), 159.6(\mathrm{~s}, \mathrm{C} 4)$, $158.8(\mathrm{~s}, \mathrm{C} 2), 157.5$ (s, C1 of $\mathrm{OPh}), 145.9(\mathrm{~s}, \mathrm{C} 8 \mathrm{a})$, 143.9 (s, C1 of Ph), 135.2 (d, C7), 129.6 (d, C3 of OPh), 127.7 (d, C3 of Ph), 127.3 (d, C5), 126.9 (d, C2 of Ph), 126.6 (d, C6), 126.4 (d, C8), 126.3 (d, C4 of Ph), 121.6 (d, C4 of OPh), $120.4(\mathrm{~s}, \mathrm{C} 4 \mathrm{a}), 115.4(\mathrm{~d}, \mathrm{C} 2$ of $\mathrm{OPh})$, $78.2(\mathrm{~d}, \mathrm{CH}), 72.1(\mathrm{~d}, \mathrm{CH}), 48.4(\mathrm{~d}, \mathrm{CH}), 26.1\left(\mathrm{t}, \mathrm{CH}_{2}\right)$, 20.5 (t, $\left.\mathrm{CH}_{2}\right), 15.5\left(\mathrm{q}, \mathrm{CH}_{3}\right), 10.5\left(\mathrm{q}, \mathrm{CH}_{3}\right)$.

\section{Compound 26b}

${ }^{1} \mathrm{H}$ NMR (DMSO- $d_{6}$ ): $\delta=11.42$ (br s, exch., $1 \mathrm{H}, \mathrm{NH}$ ), 8.13-6.97 (m, $15 \mathrm{H}, \mathrm{ArH}$ and $\mathrm{OH}), 5.34(\mathrm{~d}, J=4 \mathrm{~Hz}, 1$ $\mathrm{H}, \mathrm{CHOH}), 4.98(\mathrm{t}, J=7 \mathrm{~Hz}, 1 \mathrm{H}, \mathrm{CH}), 3.12(\mathrm{~m}, 1 \mathrm{H}$, $\mathrm{CH}), 2.14-1.38\left(\mathrm{~m}, 4 \mathrm{H}, 2 \mathrm{CH}_{2}\right), 1.09(\mathrm{t}, J=7 \mathrm{~Hz}, 3 \mathrm{H}$, $\left.\mathrm{CH}_{3}\right), 0.54\left(\mathrm{t}, J=7.5 \mathrm{~Hz}, 3 \mathrm{H}, \mathrm{CH}_{3}\right)$

${ }^{13} \mathrm{C}$ NMR (DMSO- $\left.d_{6}\right): \delta=170.7(\mathrm{~s}, \mathrm{C}=\mathrm{O}), 159.2(\mathrm{~s}, \mathrm{C} 4)$, 158.4 (s, C2), 157.5 (s, C1 of OPh), 146.3 (s, C8a), 144.0 (s, C1 of Ph), 135.0 (d, C7), 129.5 (d, C3 of OPh), 127.9 (d, C3 of Ph), 127.5 (d, C5), 127.0 (d, C2 of Ph), 126.7 (d, C6), 126.4 (d, C8), 126.3 (d, C4 of Ph), 121.7 (d, C4 of OPh), 120.6 (s, C4a), 115.7 (d, C2 of OPh), $77.3(\mathrm{~d}, \mathrm{CH}), 72.9(\mathrm{~d}, \mathrm{CH}), 50.1(\mathrm{~d}, \mathrm{CH}), 25.6\left(\mathrm{t}, \mathrm{CH}_{2}\right)$, $18.5\left(\mathrm{t}, \mathrm{CH}_{2}\right), 11.7\left(\mathrm{q}, \mathrm{CH}_{3}\right), 9.4\left(\mathrm{q}, \mathrm{CH}_{3}\right)$.

\section{2-(1-Ethyl-2-hydroxy-2-phenylpropyl)-3-(2-} phenoxybutyrylamino)quinazolin-4(3H)-one (27)

mp 105-107 ${ }^{\circ} \mathrm{C} ; \mathbf{2 7} \mathbf{a} / \mathbf{2 7 b}=4: 5\left({ }^{1} \mathrm{H}\right.$ NMR).

EI-MS: $m / z(\%)=365$ (19), 337 (25), 272 (10), 244 (53), 230 (100), 214 (24), 202 (27).

EI-MS: $m / z(\%)=486\left(\mathrm{MH}^{+}, 33\right), 366(15), 309(100)$.

HRMS: $m / z$ calcd for $\mathrm{C}_{29} \mathrm{H}_{32} \mathrm{~N}_{3} \mathrm{O}_{4}\left(\mathrm{MH}^{+}\right)$: 486.2393; found: 486.2394 .

\section{Compound 27a}

${ }^{1} \mathrm{H}$ NMR (DMSO- $d_{6}$ ): $\delta=11.34$ (br s, exch., $1 \mathrm{H}, \mathrm{NH}$ ), 8.21-6.94 (m, 14 H, ArH), 5.27 (s, exch., $1 \mathrm{H}, \mathrm{OH}), 5.06-$ $5.00(\mathrm{~m}, 1 \mathrm{H}, \mathrm{CH}), 3.44-3.41(\mathrm{~m}, 1 \mathrm{H}, \mathrm{CH}), 2.15-1.56$ (m, $\left.4 \mathrm{H}, 2 \mathrm{CH}_{2}\right), 1.19\left(\mathrm{~s}, 3 \mathrm{H}, \mathrm{CH}_{3}\right), 0.85$ (t, $J=7 \mathrm{~Hz}, 3$ $\left.\mathrm{H}, \mathrm{CH}_{3}\right), 0.23$ (t, $J=7.5 \mathrm{~Hz}, 3 \mathrm{H}, \mathrm{CH}_{3}$ ).

${ }^{13} \mathrm{C}$ NMR (DMSO- $\left.d_{6}\right): \delta=172.2(\mathrm{~s}, \mathrm{C}=\mathrm{O}), 160.0(\mathrm{~s}, \mathrm{C} 4)$, 158.4 (s, C2), 157.5 (s, C1 of OPh), 147.0 (s, C8a), 145.7 (s, C1 of Ph), 135.3 (d, C7), 129.7 (d, C3 of OPh), 127.9 (d, C3 of Ph), 127.4 (d, C5), 127.2 (d, C6), 126.6 (d, C8), 124.7 (d, C2 of Ph), 121.5 (d, C4 of Ph), 120.3 (d, C4 of OPh), 119.4 (s, C4a), 115.1 (d, C2 of OPh), $76.9(\mathrm{~d}, \mathrm{CH}), 75.3(\mathrm{~s}, \mathrm{C}-\mathrm{OH}), 50.2(\mathrm{~d}, \mathrm{CH}), 29.5$ (q, $\left.\mathrm{CH}_{3}\right), 25.9\left(\mathrm{t}, \mathrm{CH}_{2}\right), 21.9\left(\mathrm{t}, \mathrm{CH}_{2}\right), 11.9\left(\mathrm{q}, \mathrm{CH}_{3}\right), 9.7$ (q, $\left.\mathrm{CH}_{3}\right)$.

\section{Compound 27b}

${ }^{1} \mathrm{H}$ NMR (DMSO- $d_{6}$ ): $\delta=11.34$ (br s, exch., $1 \mathrm{H}, \mathrm{NH}$ ), 8.21-6.94 (m, $14 \mathrm{H}, \mathrm{ArH}), 6.01$ (s, exch., $1 \mathrm{H}, \mathrm{OH}$ ), 5.06$5.00(\mathrm{~m}, 1 \mathrm{H}, \mathrm{CH}), 3.10-3.08(\mathrm{~m}, 1 \mathrm{H}, \mathrm{CH}), 2.15-1.56$ $\left(\mathrm{m}, 4 \mathrm{H}, 2 \mathrm{CH}_{2}\right), 1.28\left(\mathrm{~s}, 3 \mathrm{H}, \mathrm{CH}_{3}\right), 1.15(\mathrm{t}, J=7 \mathrm{~Hz}, 3$ $\left.\mathrm{H}, \mathrm{CH}_{3}\right), 0.33\left(\mathrm{t}, J=7.5 \mathrm{~Hz}, 3 \mathrm{H}, \mathrm{CH}_{3}\right)$.

${ }^{13} \mathrm{C}$ NMR (DMSO- $\left.d_{6}\right): \delta=170.6(\mathrm{~s}, \mathrm{C}=\mathrm{O}), 162.1(\mathrm{~s}, \mathrm{C} 4)$, 158.6 (s, C2), 157.6 (s, C1 of OPh), 146.7 (s, C8a), 145.1 (s, C1 of Ph), 135.5 (d, C7), 129.5 (d, C3 of OPh), 128.0 (d, C3 of Ph), 127.5 (d, C5), 127.1 (d, C6), 126.5 (d, C8), 125.1 (d, C2 of Ph), 121.5 (d, C4 of Ph), 120.6 (d, C4 of OPh), 120.4 (s, C4a), 115.4 (d, C2 of OPh), $78.2(\mathrm{~d}, \mathrm{CH}), 75.5(\mathrm{~s}, \mathrm{C}-\mathrm{OH}), 52.1(\mathrm{~d}, \mathrm{CH}), 30.8(\mathrm{q}$, $\left.\mathrm{CH}_{3}\right), 26.5\left(\mathrm{t}, \mathrm{CH}_{2}\right), 23.6\left(\mathrm{t}, \mathrm{CH}_{2}\right), 11.3\left(\mathrm{q}, \mathrm{CH}_{3}\right), 9.5(\mathrm{q}$, $\left.\mathrm{CH}_{3}\right)$.

\section{Acknowledgment}

We thank the EPSRC Mass Spectroscopy Service, University of Wales Swansea, for recording the mass spectra. We also thank the EPSRC, the Higher Education Funding Council for Wales (ELWaHEFCW) and the University of Wales Swansea for grants that enabled the purchase and upgrading of NMR equipment used in the course of this work. G. A. El-Hiti thanks the University of Wales Swansea for financial support and the Royal Society of Chemistry for an international author grant.

\section{References}

(1) Permanent address; Department of Chemistry, Faculty of Science, Tanta University, Tanta 31527, Egypt.

(2) (a) Beak, P.; Zajdel, W. J.; Reitz, D. B. Chem. Rev. 1984, 84, 471. (b) Snieckus, V. Chem. Rev. 1990, 90, 879. (c) ElHiti, G. A. Heterocycles 2000, 53, 1839. (d) Mongin, F.; Quéguiner, G. Tetrahedron 2001, 57, 4059. (e) Turck, A.; Plé, N.; Mongin, F.; Quéguiner, G. Tetrahedron 2001, 57, 4489. (f) Anctil, E. J.-G.; Snieckus, V. J. Organomet. Chem. 2002, 653, 150. (g) Smith, K.; El-Hiti, G. A. Curr. Org. Synth. 2004, 1, in press. (h) Chinchilla, R.; Nájera, C.; Yus, M. Chem. Rev. 2004, in press.

(3) See for example: (a) Choppin, S.; Gros, P.; Fort, Y. Org. Lett. 2000, 2, 803. (b) Kondo, Y.; Asai, M.; Miura, T.; Uchiyama, M.; Sakamoto, T. Org. Lett. 2001, 3, 13. (c) Rebstock, A.-S.; Mongin, F.; Trécourt, F.; Quéguiner, G. Tetrahedron Lett. 2002, 43, 767. (d) Rhonnstad, P.; Wensbo, D. Tetrahedron Lett. 2002, 43, 3137. (e) Mukherjee, C.; De, A. Synlett 2002, 325. (f) Cuperly, D.; Gros, P.; Fort, Y. J. Org. Chem. 2002, 67, 238. (g) Gros, P.; Louërat, F.; Fort, Y. Org. Lett. 2002, 4, 175. (h) Toudic, F.; Plé, N.; Turck, A.; Quéguiner, G. Tetrahedron 2002, 58, 238. (i) 
Bunch, L.; Krogsgaard-Larsen, P.; Madsen, U. J. Org. Chem. 2002, 67, 2375. (j) Katritzky, A. R.; Manju, K.; Steel, P. J. J. Org. Chem. 2003, 68, 407. (k) Gros, P.; Choppin, S.; Fort, Y. J. Org. Chem. 2003, 68, 2243. (1) El-Hiti, G. A. Synthesis 2004, 363. (m) Cabiddu, M. C.; Cabiddu, S.; Cadoni, E.; De Montis, S.; Fattuoni, C.; Melis, S. Tetrahedron 2004, 60, 3915.

(4) Murray, T. P.; Hay, J. V.; Protlock, D. E.; Wolfe, J. F. J. Org. Chem. 1974, 39, 595.

(5) Rathman, T. L.; Sleevi, M. C.; Krafft, M. F.; Wolfe, J. F. J. Org. Chem. 1980, 45, 2169.

(6) Smith, K.; El-Hiti, G. A.; Abdel-Megeed, M. F.; Abdo, M. A. Collect. Czech. Chem. Commun. 1999, 64, 515.

(7) Smith, K.; El-Hiti, G. A.; Abdel-Megeed, M. F.; Abdo, M. A. J. Org. Chem. 1996, 61, 656.

(8) El-Hiti, G. A. Monatsh. Chem. 2004, 135, 323.

(9) Smith, K.; El-Hiti, G. A.; Abdel-Megeed, M. F.; Abdo, M. A. J. Org. Chem. 1996, 61, 647.

(10) Smith, K.; El-Hiti, G. A.; Abdo, M. A.; Abdel-Megeed, M. F. J. Chem. Soc. Perkin Trans. 1 1995, 1029.

(11) Plé, N.; Turck, A.; Chapoulaud, V.; Quéguiner, G. Tetrahedron 1997, 53, 2871.

(12) Dai, X.; Virgil, S. Tetrahedron Asymmetry 1999, 10, 25.

(13) Chapoulaud, V. G.; Salliot, I.; Plé, N.; Turck, A.; Quéguiner, G. Tetrahedron 1999, 55, 5389.

(14) See for example: (a) Honda, G.; Tabata, M.; Tsuda, M. Planta Med. 1979, 37, 172. (b) Johne, S. Prog. Drug Res. 1982, 26, 259. (c) Johne, S. The Alkaloids 1986, 29, 99. (d) Schlecker, R.; Treiber, H. J.; Behl, B.; Hofmann, H. P. Ger. Offen 4241 563, 1994; Chem. Abstr. 1994, 121, 230787. (e) Barker, A. J. Eur. Patent 635 498, 1995; Chem. Abstr. 1995, 122, 214099. (f) Barker, A. J.; Johnstine, C. PCT Int Appl WO 300 44, 1997; Chem. Abstr. 1995, 127, 220671. (g) Hour, M.-J.; Huang, L.-J.; Kuo, S.-C.; Xia, Y.; Bastow, K.; Nakanishi, Y.; Hamel, E.; Lee, K.-H. J. Med. Chem. 2000, 43, 4479. (h) Ho, N.; Harapanhalli, R. S.; Dahman, B. A.; Chen, K.; Wang, K. Bioconjugate Chem. 2002, 13, 357.

(15) See for example: (a) Smith, K.; El-Hiti, G. A.; Hamilton, A. J. Chem. Soc., Perkin Trans. 1 1998, 4041. (b) Smith, K.; El-Hiti, G. A.; Pritchard, G. J.; Hamilton, A. J. Chem. Soc., Perkin Trans. 1 1999, 2299. (c) Smith, K.; El-Hiti, G. A.; Shukla, A. P. J. Chem. Soc., Perkin Trans. 1 1999, 2305. (d) Smith, K.; El-Hiti, G. A.; Abdel-Megeed, M. F. Russ. J. Org. Chem., 2003, 39, 430. (e) Smith, K.; El-Hiti, G. A.; Hawes, A. C. Synthesis 2003, 2047. (f) Smith, K.; El-Hiti, G. A.; Mahgoub, S. A. Synthesis 2003, 2345. (g) El-Hiti, G. A. Synthesis 2003, 2799.

(16) Sammour, A.; Selim, M.; El Kasaby, M.; Abdalla, M. Acta Chim. (Budapest) 1973, 78, 293; Chem. Abstr. 1973, 79, 137061.

(17) (a) Essawy, A.; El-Hashash, M. A.; El-Gendy, A. M.; Hamad, M. M. M. Indian J. Chem. 1982, 21B, 593.

(18) El-Hiti, G. A. Spectrosc. Lett., 1999, 32, 671.

(19) Vogel's Textbook of Practical Organic Chemistry: 5th Edn, Longman, Harlow 1989.

(20) Perrin, D. D.; Armarego, W. L. F.; Perrin, D. R. Purification of Laboratory Chemicals, Pergamon, 3rd Edn, Butterworth Heinemann, Oxford, 1988. 


\section{Lithiation of Chiral 3-Acylamino-2-alkylquinazolin-4(3H)-ones}

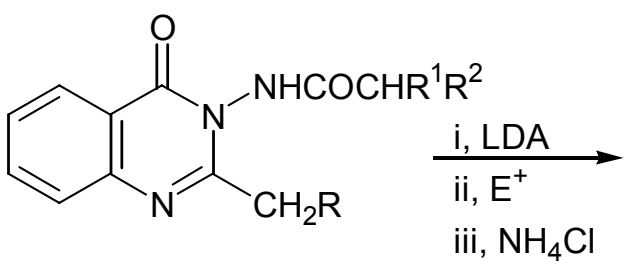<smiles>[R]C(=O)Nn1c(C([R])F)nc2ccccc2c1=O</smiles>

\section{Manuscript submission checklist}

- Statement of significance of work.

- Full mailing address, telephone and fax numbers, and email address of the corresponding author.

- Paper save as a PDF file.

- Original Word file.

- Original graphic files.

- Graphical abstract. 